\title{
DISCRETE PARTICLE SIMULATION OF BUBBLE AND SLUG FORMATION IN A TWO-DIMENSIONAL GAS-FLUIDISED BED: A HARD-SPHERE APPROACH
}

\author{
B. P. B. HOOMANS ${ }^{\dagger}$, J. A. M. KUIPERS, W. J. BRIELS and W. P. M. VAN SWAAIJ \\ Department of Chemical Engineering, Twente University of Technology, P.O. Box 217, 7500 AE Enschede, \\ The Netherlands
}

(First received 13 June 1995; accepted 11 July 1995)

\begin{abstract}
A discrete particle model of a gas-fluidised bed has been developed and in this the twodimensional motion of the individual, spherical particles was directly calculated from the forces acting on them, accounting for the interaction between the particles and the interstitial gas phase. Our collision model is based on conservation laws for linear and angular momentum and requires, apart from geometrical factors, two empirical parameters: a restitution coefficient and a friction coefficient. A sequence of collisions is processed using techniques which find their application in hard-sphere simulations which are commonly encountered in the field of molecular dynamics. The hydrodynamic model of the gas phase is based on the volume-averaged Navier-Stokes equations. Simulations of bubble and slug formation in a small twodimensional bed (height $0.50 \mathrm{~m}$, width $0.15 \mathrm{~m})$ with 2400 particles $\left(d_{p}=4 \mathrm{~mm}\right.$, material: aluminium, $\rho=$ $2700 \mathrm{~kg} \mathrm{~m}^{-3}$ ) showed a strong dependency of the flow behaviour with respect to the restitution and friction coefficient. A preliminary experimental validation of our model was performed using a small scale "two-dimensional" gas-fluidised bed (height $0.30 \mathrm{~m}$, width $0.15 \mathrm{~m}$, depth $0.015 \mathrm{~m}$ ) with $850 \mu \mathrm{m}$ ballotini glass particles $\left(\rho=2930 \mathrm{~kg} \mathrm{~m}^{-3}\right)$ as the bed material. Results compared fairly well with the results of a simulation which was performed with 40,000 particles using realistic values for the restitution and friction coefficients which were obtained from simple independent experiments.
\end{abstract}

\section{INTRODUCTION}

Two-fluid models have been frequently used to describe hydrodynamic phenomena in fluidised beds. There are several examples of such models in the literature with most recent contributions by, among others, Ding and Gidaspow (1990), Kuipers et al. (1992) and Nieuwland (1994). In the two-fluid models the gas and the solids phase are considered to be continuous and fully interpenetrating. Both phases are described in terms of separate sets of conservation equations with appropriate interaction terms representing the coupling between the phases. Though physical characteristics of the solid particles such as the shape and size are included in the continuum representation through the empirical relations for the interfacial friction, these models do not recognise the discrete character of the solid phase. In the two-fluid concept assumptions need to be made concerning the solids rheology where often Newtonian behaviour is assumed in absence of more detailed knowledge (Kuipers et al., 1992). In discrete particle simulations these assumptions need not be made since the motion of each single particle is directly calculated while accounting for interactions with other particles and the continuous phase. In fact these simulations, which can be called granular dynamics (GD) simulations, enable the calculation of transport coefficients like

\footnotetext{
${ }^{\dagger}$ Corresponding author. Email: b.p.b.hoomans@ct.utwente.nl
}

effective viscosity and self-diffusion as is done in molecular dynamics (MD) since all information is readily available (Walton, 1984). There are two essential differences between GD and MD: in a granular system energy is dissipated due to inelastic particle interactions and particle motion is induced by external forces acting on the system (i.e. gravity and drag). A limiting factor in discrete particle models is the number of particles which can be handled in a simulation. Although computing power is still increasing significantly, and rapid progress may be made by parallel computing, it is not believed that a discrete particle simulation of a complete fluidised bed of industrial scale will be possible in the near future. A discrete particle model however provides a powerful tool to obtain substantial insight in the bed hydrodynamics and by adapting techniques from MD it should be possible to arrive at improved constitutive equations for continuum models. Furthermore it is relatively easy to take a particle size distribution into account which is far more difficult, if not impossible, within the framework of the two-fluid concept. However, in the present study we focus on bubble and slug formation in a two-dimensional gas-fluidised bed with uniform particles. The modelling approach adopted in this study is partially based on techniques used in hardsphere simulations which are well known in MD. From the very beginning of fluidised bed engineering it was recognised that fluidised beds show liquid-like behaviour and from this point of view it seems a logical step to try to model a fluidised bed in a similar way 
as a hard-sphere liquid is modelled in MD. In fact a first step toward this approach was made by researchers who employed kinetic theories into their two-fluid models, as was done by, among others, Ding and Gidaspow (1990) and Nieuwland (1994).

\section{GRANULAR DYNAMICS MODELLING}

Over the last decade GD models have received increasing attention since computer facilities have reached a level of sophistication which enables this type of model to be run within reasonable CPU time constraints. The modelling approaches adopted in GD, like in MD, can be roughly divided into two groups: soft particle and hard particle approaches.

\subsection{Soft particle approaches}

In the majority of the GD models presented in the literature a soft particle approach is adopted. In this approach the motion of the particles is calculated by means of numerical integration of the Newtonian equations of motion. Apart from external forces acting on the system, the interparticle forces are of key importance to these models. In Cundall and Strack's (1979) distinct element method (DEM) the interparticle contact forces are calculated on the basis of simple mechanical models such as springs, dashpots and friction sliders. DEM has been employed by many researchers in the field of GD [Tsuji et al. (1992) and Campbell and Potapov (1993) among many others] and was also adopted by Tsuji et al. (1993) in their discrete particle simulation of a gasfluidised bed. Langston et al. (1994) used a continuous normal interaction in their work concerning granular flow in hoppers. Recently they extended their model to three dimensions (Langston et al., 1995) and they made a comparison between three forms of normal interaction where apart from a spring and a continuous interaction also a Hertzian normal interaction was considered. A characteristic feature of the soft particle models is that they are capable of handling multiple particle contacts which is of importance when modelling quasi-static systems. However, if the normal interactions between the particles are taken to be as stiff as can be derived from the particle characteristics (i.e. elastic modulus), a very small time step $\left(<10^{-6} \mathrm{~s}\right)$ is required. This constraint arises from stability considerations: the time step should be smaller than the duration of a contact. Therefore in most soft-sphere models the duration of a contact is artificially increased by allowing for softer interaction and hence reducing the required CPU time. This was also done by Tsuji et al. (1993) in their simulation of a gas-fluidised bed where they assumed that the effect of this softer interaction on the particle motion is only secondary.

\subsection{Hard particle approaches}

In hard particle models the interaction forces are assumed to be impulsive and hence the particles only exchange momentum by means of collisions. Hogue and Newland (1994) presented a two-dimensional model where the particle interaction is based on binary, quasi-instantaneous collisions. Their model is capable of handling particles of arbitrary geometry using a polar representation of the particle shape. The collision model of Wang and Mason (1992) is used to compute the dynamics of inelastic collisions with friction. Due to the complexity of the system an idealised motion update scheme is used which causes an error in the time-evolution of the system configuration.

In this work only spherical particles are considered and therefore it has been possible to develop a model which combines a simplified version of the collision model of Wang and Mason (1992) with techniques which are used in hard-sphere simulations which are commonly encountered in MD (Allen and Tildesley, 1990). A characteristic feature of a hard-sphere simulation is that a sequence of collisions is processed, one collision at a time, assuming instantaneous collisions. In our model two time scales can be distinguished which means that two different time steps are used. We use a constant time step DT to account for the external forces acting on the particles and within this time step we perform a hard-sphere simulation. In this hard-sphere simulation the time step for the integration of the particle motion is calculated within the model as will be explained in Section 2.4. An important feature of our hard-sphere model is that simulations can actually be performed with realistic values for the key parameters (restitution and friction coefficients) whilst in soft particle approaches softer normal interactions have to be assumed.

\subsection{Collision dynamics}

The main assumptions of the collision model are concerned with the particle shape, the deformation history during the collision and the nature of the collisions and can be summarised as follows:

- particles are spherical and quasi rigid, the shape is retained after impact,

- collisions are binary and quasi-instantaneous,

- contacts occur at a point,

- motion is two-dimensional with the particle centres of mass moving in one plane,

- particles are in free flight in between collisions,

- interaction forces are impulsive and all other finite forces are negligible during collision.

These assumptions are believed to be realistic for collisions that occur in the fluidisation of relatively coarse particles $(>100 \mu \mathrm{m})$, say typically B and $D$ powders according to the classification of Geldart (1973). Similar assumptions concerning the nature of the collisions are found in the works of other researchers in this field (Buyevich, 1994; Nieuwland, 1994).

The collision model used in this work mainly follows the methodology presented by Wang and Mason (1992). The collision coordinate system used to describe the collision dynamics is defined in Fig. 1. 


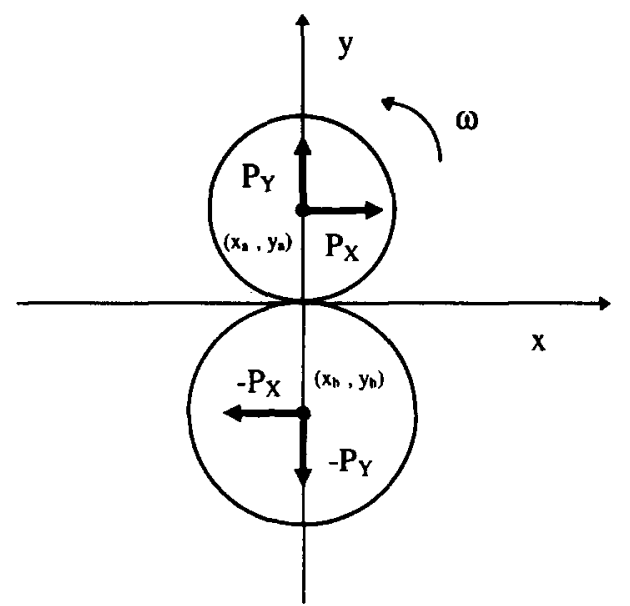

Fig 1. Collision coordinate system. $P_{X}$ and $P_{Y}$ are the components of impulse acting on particle $a$, as defined in eq. (7).

When two particles collide, impulses in the normal direction $P_{Y}$ and in the tangential direction $P_{X}$ at the contact point are produced and these will change the motions of the particles. In the collision coordinate system the origin is chosen at the point of contact denoted at times by subscript $\mathrm{cp}$, the $y$-axis points along the normal direction and the $x$-axis points along the tangential direction. The collision coordinate system can easily be constructed from the position vectors in system coordinates. At the beginning of the contact the particles $a$ and $b$ have initial linear and angular velocities indicated with the subscript 0 . At any instant during the impact, the motions of the particles are governed by the linear and angular impulse-momentum laws which yields the following set of equations for a binary collision of two spheres, $a$ and $b$, moving in a two-dimensional $x y$-space:

$$
\begin{aligned}
m_{a}\left(v_{x, a}-v_{x, a 0}\right) & =P_{X} \\
m_{a}\left(v_{y, a}-v_{y, a 0}\right) & =P_{Y} \\
I_{a}\left(\omega_{a}-\omega_{a 0}\right) & =P_{X} y_{a} \\
m_{b}\left(v_{x, b}-v_{x, b 0}\right) & =-P_{X} \\
m_{b}\left(v_{y, b}-v_{y, b 0}\right) & =-P_{Y} \\
I_{b}\left(\omega_{b}-\omega_{b 0}\right) & =-P_{X} y_{b}
\end{aligned}
$$

where $m_{a}$ is the mass of particle $a, I_{a}$ is the moment of inertia of particle $a$, defined by $I=m R_{\mathrm{gyr}}^{2}$, where $R_{\mathrm{gyr}}$ is the radius of gyration of the particle (for spherical particles: $R_{\mathrm{gyr}}^{2}=\frac{2}{5} R_{p}^{2}$ ). The components of impulse in eqs (1) and (2) can be obtained from the integration of the force exerted on particle $a$ by particle $b$ during the contact:

$$
\bar{P}(t)=\int_{0}^{t} \bar{F}_{a b}(\tau) \mathrm{d} \tau .
$$

Equations (4) and (5) are obtained in a similar way using the principle of action equals - reaction. Equa- tions (3) and (6) follow from conservation of angular momentum for each particle. The impact process is analysed further in terms of a slip velocity $S$ and a compression velocity $C$ (tangential and normal components of the relative velocity at the contact point, respectively) which are defined as follows:

$$
\begin{aligned}
& S \equiv v_{x, a c p}-v_{x, b c p} \\
& C \equiv v_{y, a c p}-v_{y, b c p} .
\end{aligned}
$$

From the definitions (8) and (9) and eqs (1)-(6) the following expressions can be derived for the slip velocity and the compression velocity during impact:

$$
S=S_{0}+B_{1} P_{X}
$$

where

$$
\begin{aligned}
& S_{0}=\left[v_{x, a 0}+\left(\omega_{a 0} R_{a}\right)\right]-\left[v_{x, b 0}-\left(\omega_{b 0} R_{b}\right)\right] \\
& B_{1}=\frac{1}{m_{a}}+\frac{1}{m_{b}}+\frac{R_{a}^{2}}{I_{a}}+\frac{R_{b}^{2}}{I_{b}}
\end{aligned}
$$

and

$$
C=C_{0}+B_{2} P_{Y}
$$

where

$$
\begin{aligned}
& C_{0}=v_{y, a 0}-v_{y, b 0} \\
& B_{2}=\frac{1}{m_{a}}+\frac{1}{m_{b}} .
\end{aligned}
$$

$S_{0}$ and $C_{0}$ are the initial slip and compression velocities, respectively, and $B_{1}$ and $B_{2}$ are collision constants. In the case of a collision with a system wall, the wall is taken to be particle $b$ with velocities set equal to zero and the mass set equal to infinity. From eqs (1)-(6) the linear and angular velocities of both particles can be calculated in collision coordinates when the values for $P_{X}$ and $P_{Y}$ at the end of the collision process are known. In order to be able to calculate these values, which are denoted by $\hat{P}_{X}$ and $\hat{P}_{Y}$, respectively, constitutive equations for restitution and friction are needed. Newton's law of restitution relates the compression velocity at the end of the collision process to the initial compression velocity:

$$
e=-\hat{C} / C_{0}
$$

where $C_{0}$ is negative. The changes in the normal and tangential components of the impulse during sliding are related by Coulomb's law of friction:

$$
\frac{\mathrm{d} P_{X}}{\mathrm{~d} P_{Y}}=-s \mu
$$

where, $s=\operatorname{sign}\left(S_{0}\right)=S_{0} /\left|S_{0}\right|$, with $S_{0}$ being the initial sliding velocity.

In this work, both the restitution and the friction coefficient are assumed to be constant, which is a simplifying assumption. We do however distinguish between restitution and friction coefficients for particle particle collisions and particle-wall collisions, indicated with the subscript $w$. 
The energy dissipated during a collision consists of contributions by the tangential and the normal components:

$$
E_{d s p}=E_{d s p, X}+E_{d s p, Y}
$$

where

$$
\begin{aligned}
E_{d s p, X} & =\int_{0}^{\hat{f}_{X}} S \mathrm{~d} P_{X} \\
E_{d s p, Y} & =\int_{0}^{\hat{f}_{Y}} C \mathrm{~d} P_{Y} .
\end{aligned}
$$

For spherical particles the normal component of impulse is not affected by the tangential component. An expression for the normal component of impulse $\hat{P}_{Y}$ can be derived starting with eq. (13) at the end of collision:

$$
\hat{C}=C_{0}+B_{2} \hat{P}_{Y} .
$$

Substitution of Newton's law leads to the following expression for $\hat{P}_{Y}$ :

$$
\hat{P}_{Y}=-(1+e) C_{0} / B_{2} .
$$

The energy dissipated in the collision by the normal component is in this case given by

$$
E_{d s p, Y}=\frac{C_{0}^{2}}{2 B_{2}}\left(1-e^{2}\right) .
$$

For the tangential component two classes of impact have to be distinguished, sliding and sticking. The class of impact can be determined as follows:

sliding:

$$
\left|S_{0}\right| \geqslant \mu B_{1} \hat{P}_{Y}
$$

sticking:

$$
\left|S_{0}\right|<\mu B_{1} \hat{P}_{Y} .
$$

For collisions of the sliding class the slip velocity never becomes zero; hence Coulomb's law applies to the whole period of collision. The tangential component of impulse is then given by

$$
\hat{P}_{X}=-\mu s \hat{P}_{Y}
$$

and the amount of energy dissipated during collision by this component follows from

$$
E_{d s p, X}=-\mu(1+e) \frac{C_{0}}{B_{2}}\left[\left|S_{0}\right|+\frac{\mu B_{1}(1+e) C_{0}}{2 B_{2}}\right] .
$$

For collisions of the sticking class the frictional forces are sufficiently high in order to terminate sliding during collision. This means that the slip velocity equals zero at the end of collision and the tangential component of impulse is given by

$$
\hat{P}_{X}=-S_{0} / B_{1}
$$

and the amount of energy dissipated by the tangential component is for the sticking class given by

$$
E_{d s p, X}=S_{0}^{2} / 2 B_{1} \text {. }
$$

\subsection{Sequence of collisions}

In our model we use a constant time step DT to account for the forces acting on the particles. Within this time step the velocities are assumed to change only due to collisions and a sequence of collisions is processed one collision at a time. In order to do so it is necessary to determine which pair of particles will collide first. Therefore it is necessary to determine the collision times of all relevant collision pairs. The collision time $t_{a b}$ of a collision pair $a, b$ (where $a$ and $b$ refer to index numbers; each particle in the simulation can be identified by its index number) is defined as the time interval until collision. It can be calculated from the initial position and velocity vectors of both particles which are shown in Fig. 2. When particles $a$ and $b$ collide the distance between the two centres of mass is equal to the sum of the two radii. This yields a quadratic equation in $t_{a b}$ the smallest solution of which corresponds to collision (Allen and Tildesly, 1990):

$t_{a b}=\frac{-\mathbf{r}_{a b} \cdot \mathbf{v}_{a b}-\sqrt{\left(\mathbf{r}_{a b} \cdot \mathbf{v}_{a b}\right)^{2}-v_{a b}^{2}\left[r_{a b}^{2}-\left(R_{a}+R_{b}\right)^{2}\right]}}{v_{a b}^{2}}$,

where $\mathbf{r}_{a b} \equiv \mathbf{r}_{a}-\mathbf{r}_{b}$ and $\mathbf{v}_{a b} \equiv \mathbf{v}_{a}-\mathbf{v}_{b}$. Note that if $\mathbf{r}_{a b} \cdot \mathbf{v}_{a b}>0$ the particles are moving away from each other and will not collide. In case of a collision with a wall the collision time follows simply from the distance to the wall and the normal velocity component toward that wall which leads for a vertical wall to the following expression:

$$
t_{a, \text { wall }}=\frac{\left(\left|x_{w_{a} a 11}\right|+R_{a}\right)-\left|\mathbf{r}_{x, a}\right|}{v_{x, a}} .
$$

The algorithm used to process a sequence of collisions within a constant time step DT is presented in Fig. 3. First the collision lists are initialised in which for each particle a collision partner and a corresponding collision time are stored. For each particle the smallest collision time is determined by scanning all relevant collision partners for a possible collision. For particle $a$, only the particles $b$ with a higher index number $(b>a)$ are checked in order to limit the number of collision pairs to be checked. The variable "acctim"

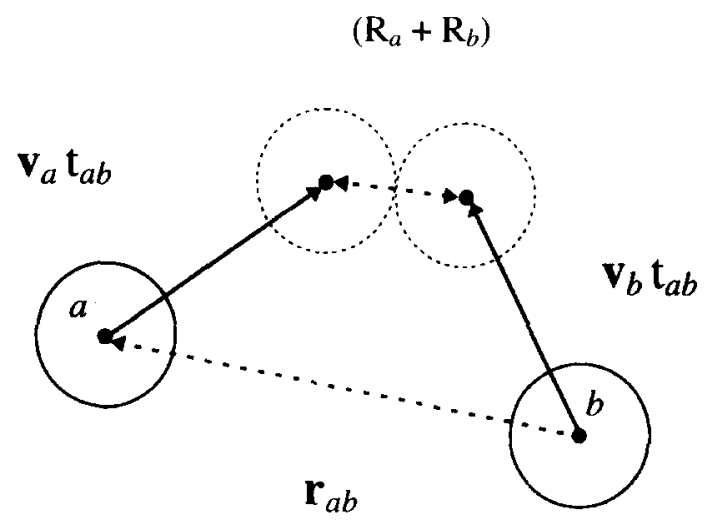

Fig 2. Determination of the collision time. 


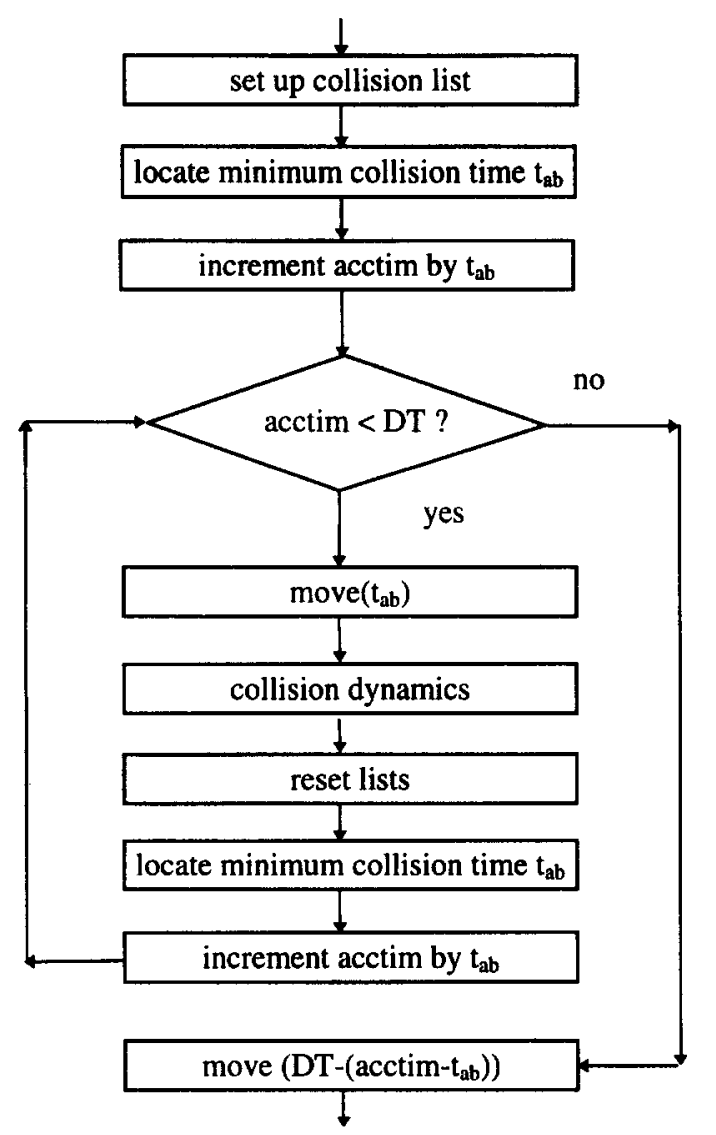

Fig 3. Algorithm for the processing of a sequence of collisions within a constant time step DT.

keeps track of the time spent since the beginning of the time step. As soon as a minimum collision time is found that after addition to "acctim" is greater than the time step DT, the loop is finished. After the loop is finished the particles have to be moved forward until "acctim" equals DT. During this motion no collision occurs. In the routine "move" the collision times of all particles are reduced with $t_{a b}$ and the particle positions are updated using a simple explicit integration:

$$
\mathbf{r}_{a}\left(t+t_{a b}\right)=\mathbf{r}_{a}(t)+\mathbf{v}_{a} t_{a b}
$$

The calculation of the collision dynamics involves a transformation to collision coordinates as well as a back-transformation to system coordinates. Subsequently the routine "reset lists" is entered where new collision times and partners have to be found for the particles that just collided and the particles that were about to collide with either $a$ or $b$. Finally a new collision pair has to be detected and "acctim" can be incremented with the new collision time $t_{a b}$.

Substantial CPU time can be saved by employing a nearest neighbour list. In the neighbour list of particle $a$ (Fig. 4), all the particles which are found within a square of size $D_{\text {nblist }}$ with particle $a$ located at the centre, are stored. When looking for a collision partner for particle $a$ only the particles in the neighbour list need to be scanned. In Fig. 4 particle $a$ is coloured

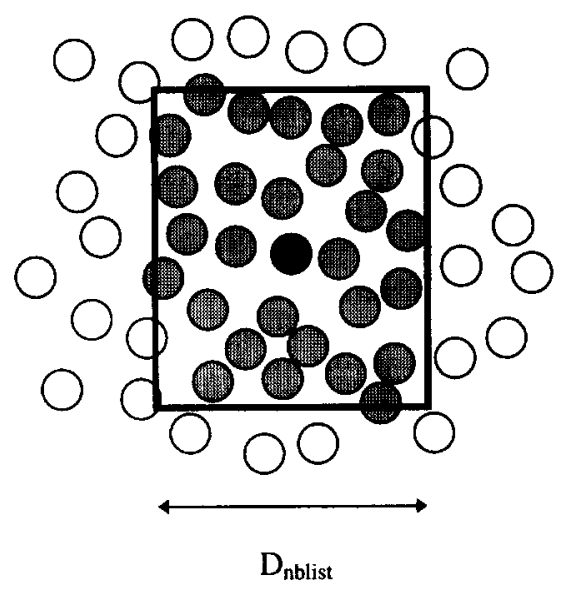

Fig 4. The principle of a square neighbour list, the shaded particles are stored in the neighbour list of the black particle.

black and its neighbour particles are shaded. There is an optimum in the choice of $D_{n b l i s t}$ but we choose $D_{\text {nblist }}$ to be 5 times the particle diameter $d_{p}$. In most MD-codes neighbour lists are constructed by defining a cut-off distance $R_{\text {nblist }}$ which gives a circular area (or a spherical space in the three-dimensional case) in which the neighbour particles are found. Since this involves the computation of squares which goes at cost of CPU time, a square neighbour list version is used in our model. The neighbour lists are updated at each time step $d t_{\text {nblist }}$. In a regular simulation $d t_{\text {nblist }}$ was chosen to be $10^{-3}$ (s) which is 4 to 10 times the time step used to calculate the influence of the force field (DT). When updating the neighbour list it is still not necessary to scan all particles. The solution of the gas flow field (Section 3) requires the computational domain to be divided into cells; for each cell the particles which centre of mass can be found in that cell are stored in a list. When updating the neighbour list, only the cell where the particle centre of mass is found and the three nearest adjacent cells are scanned for possible neighbours. A similar procedure was recently presented by Langston et al. (1995).

\subsection{Forces acting on a particle}

The particle velocities are updated using a simple explicit integration formula:

$$
\mathbf{v}_{p}=\mathbf{v}_{p}^{*}+\frac{d \mathbf{v}_{p}}{\mathrm{~d} t} \mathrm{DT} .
$$

The new particle velocity is obtained from the most recent value $\left(v_{p}^{*}\right)$ to which the acceleration multiplied by the time step is added. During the time step the velocity could have changed due to collisions which narrows the spectrum of integration techniques that can be used which also applies to eq. (32). For each particle the acceleration is calculated from a force balance over that particle:

$$
\frac{\mathrm{dv}_{p}}{\mathrm{~d} t}=\mathbf{g}+\frac{\mathbf{F}_{\mathbf{d}}}{m_{p}}
$$


where $\mathbf{g}$ is the gravitational acceleration vector, $\mathbf{F}_{d}$ is the drag force exerted on the particle and $m_{p}$ is the particle mass. Note that in the force balance the interparticle forces have been neglected, which is allowed for the relatively coarse particles used in our simulations, as well as the buoyancy force which is allowed because of the negligible density of the gas phase with respect to the density of the solids (Richardson and Zaki, 1954). The drag force on a suspended particle is given by

$$
\mathbf{F}_{d}=\frac{1}{8} \pi d_{p}^{2} C_{d}^{\prime} \rho_{g}|\mathbf{v}| \mathbf{v}
$$

where $\mathbf{v}$ in $|\mathbf{v}| \mathbf{v}$ denotes the relative superficial gas velocity vector, hence

$$
|\mathbf{v}| \mathbf{v}=\varepsilon^{2}\left|\mathbf{u}-\mathbf{v}_{\boldsymbol{p}}\right|\left(\mathbf{u}-\mathbf{v}_{p}\right) .
$$

The effective drag coefficient $C_{d}^{\prime}$ depends strongly on the local void fraction (porosity, $\varepsilon$ ) in the vicinity of the particle under consideration. According to Wen and $\mathrm{Yu}(1966)$ it can be written as the product of the drag coefficient for an isolated particle and a correction factor that depends only on the porosity:

$$
C_{d}^{\prime}=C_{d} \varepsilon^{-4.7} \text {. }
$$

The drag coefficient for an isolated particle depends on the particle Reynolds number as given by Rowe and Henwood (1961):

$$
C_{d}= \begin{cases}\frac{24}{R e_{p}}\left(1+0.15\left(R e_{p}\right)^{0.687}\right), & R e_{p}<1000 \\ 0.44, & R e_{p} \geqslant 1000\end{cases}
$$

where the particle Reynolds number is defined as

$$
R e_{p}=\frac{\varepsilon \rho_{g}\left|\mathbf{u}-\mathbf{v}_{p}\right| d_{p}}{\mu_{g}} .
$$

\subsection{Gridmapping: staggered to Lagrangian}

For the calculation of the forces acting on a suspended particle, local averaged values of the pressure
( $p$, necessary in the calculation of the gas-phase density), the porosity $(\varepsilon)$ and the fluid $x$ - and $y$-velocity components $\left(u_{x}\right.$ and $\left.u_{y}\right)$ at the position of the particle (i.e. Lagrangian grid) are required. Due to the numerical solution method these variables are only known at discrete nodes in the computational domain. An area weighted averaging technique is used to obtain the local averaged value $\bar{Q}$ of a quantity $Q(i, j)$ from the four surrounding computational nodes as is shown in Fig. 5. The local averaged value can be calculated as follows:

$$
\bar{Q}=\frac{A_{i, j} Q_{i, j}+A_{i i, j} Q_{i i, j}+A_{i i, j j} Q_{i i, j j}+A_{i, j j} Q_{i, j j}}{\mathrm{DXDY}}
$$

where

$$
\begin{aligned}
A_{i, j} & =\left(\mathrm{DX}-\delta_{x}\right)\left(\mathrm{DY}-\delta_{y}\right) \\
A_{i i, j} & =\delta_{x}\left(\mathrm{DY}-\delta_{y}\right) \\
A_{i i, j j} & =\delta_{x} \delta_{y} \\
A_{i, j j} & =\left(\mathrm{DX}-\delta_{x}\right) \delta_{y} .
\end{aligned}
$$

The distances $\delta_{x}$ and $\delta_{y}$, necessary in this averaging technique, are calculated from the position of the particle in the staggered grid.

\section{GAS-PHASE HYDRODYNAMICS}

\subsection{Governing equations}

The calculation of the gas motion mainly follows the lines presented by Kuipers et al. (1992). It is based on the numerical solution of the following set of partial differential equations which can be seen as a generalised form of the Navier-Stokes equations for a gas interacting with a solid phase. Equations (42) and (43) show the mass and momentum conservation equations in vector form.

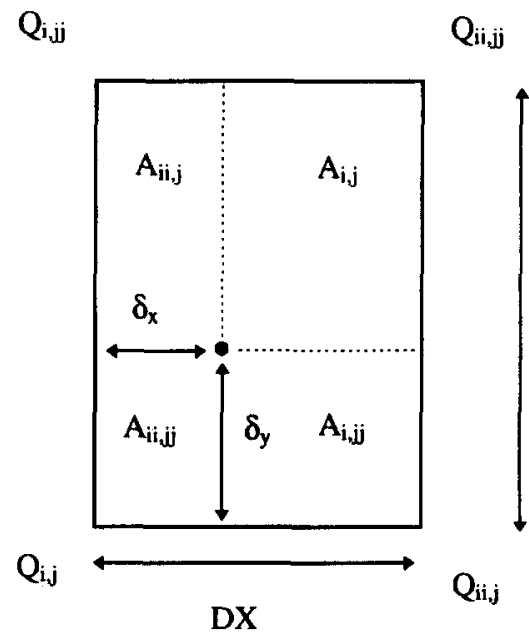

- position for the evaluation of $\bar{Q}$

DY

Fig 5. The principle of area weighted averaging. 
Continuity equation gas phase:

$$
\frac{\partial\left(\varepsilon \rho_{g}\right)}{\partial t}+\left(\nabla \cdot \varepsilon \rho_{g} \mathbf{u}\right)=0
$$

Momentum equation gas phase:

$$
\begin{aligned}
& \frac{\partial\left(\varepsilon \rho_{g} \mathbf{u}\right)}{\partial t}+\left(\nabla \cdot \varepsilon \rho_{g} \mathbf{u u}\right)=-\varepsilon \nabla p-\beta(\mathbf{u}-\mathbf{v}) \\
& -\left(\nabla \cdot \varepsilon \boldsymbol{T}_{\boldsymbol{g}}\right)+\varepsilon \rho_{g} \mathbf{g} .
\end{aligned}
$$

In this study isothermal, two-dimensional motion is considered which implies that three basic variables have to be specified. The three basic variables in the model are the pressure $(p)$ and the gas phase $x$ and $y$ velocities $\left(u_{x}\right.$ and $\left.u_{y}\right)$. The porosity $(\varepsilon)$ and the solids $x$ - and $y$-velocities $\left(v_{x}\right.$ and $\left.v_{y}\right)$ are calculated from the discrete particle model as will be explained in Section 3.4. All remaining variables have to be specified in terms of the three basic variables and/or the three variables obtained from the discrete particle model.

\subsection{Constitutive equations}

3.2.1. Gas-phase density. The gas-phase density is related to the pressure and the gas-phase temperature by the ideal gas law:

$$
\rho_{g}=\frac{M_{g}}{R T} p
$$

In our simulations the average molecular weight of air $\left(M_{g}=28.8 \times 10^{-3} \mathrm{~kg} \mathrm{~mol}^{-1}\right)$ was used and the temperature was set to a constant value of $T=293 \mathrm{~K}$.

3.2.2. Interphase momentum transfer coefficient. For porosities $\varepsilon<0.80$ the interphase momentum transfer coefficient $\beta$ has been obtained from the wellknown Ergun equation:

$$
\beta=150 \frac{(1-\varepsilon)^{2}}{\varepsilon} \frac{\mu_{g}}{d_{p}^{2}}+1.75(1-\varepsilon) \frac{\rho_{g}}{d_{p}}|\mathbf{u}-\mathbf{v}|
$$

whereas for porosities $\varepsilon \geqslant 0.80$ the following expression for the interphase momentum transfer coefficient has been used which is the correlation presented by Wen and Yu (1966) who extended the work of Richardson and Zaki (1954):

$$
\beta=\frac{3}{4} C_{d} \frac{\varepsilon(1-\varepsilon)}{d_{p}} \rho_{g}|\mathbf{u}-\mathbf{v}| \varepsilon^{-2.65} .
$$

The drag coefficient $C_{d}$ is a function of the particle Reynolds number as is given by eq. (38). The particle Reynolds number in this case is based on the cell averaged solids velocity:

$$
R e_{p}=\frac{\varepsilon \rho_{g}|\mathbf{u}-\mathbf{v}| d_{p}}{\mu_{g}} .
$$

3.2.3. Gas-phase stress tensor. The viscous stress tensor $\boldsymbol{T}_{g}$ is assumed to depend only on the gas motion. In our model the general Newtonian fluid form (Bird et al., 1960) has been implemented:

$$
\boldsymbol{T}_{g}=-\left[\left(\lambda_{g}-\frac{2}{3} \mu_{g}\right)(\nabla \cdot \mathbf{u}) \mathbf{I}+\mu_{g}\left((\nabla \mathbf{u})+(\nabla \mathbf{u})^{T}\right)\right] .
$$

In the simulations the bulk viscosity $\lambda_{g}$ was set to zero which is allowed for gases (Bird et al., 1960) whereas for the gas-phase shear viscosity a constant value of $\mu_{g}=1.8 \times 10^{-5} \mathrm{~kg} \mathrm{~m}^{-1} \mathrm{~s}^{-1}$ was taken.

\subsection{Numerical solution}

The numerical solution follows the lines of Kuipers et al. (1993) and will not be discussed in detail here. The partial differential equations for solid-phase mass and momentum conservation which were implemented in the two-fluid model developed by Kuipers et al. are no longer required in the present model. A finite differencing technique, employing a staggered grid to ensure numerical stability, is used to solve the gas-phase conservation equations. This implies that the scalar variables $(p$ and $\varepsilon$ ) are defined at the cell centre and that the velocity components are defined at the cell faces. The model is capable of performing transient two-dimensional calculations in a Cartesian or an axi-symmetrical geometry. In our simulations only the Cartesian option was used.

\subsection{Gridmapping: Lagrangian to staggered}

The solution of the Navier-Stokes equations (42) and (43) requires specification of the porosity $(\varepsilon)$ and the solids $x$ - and $y$-velocities $\left(v_{x}\right.$ and $\left.v_{y}\right)$ at the appropriate grid nodes. These values are obtained from the discrete particle model. For each particle the indices $i, j$ of the cell where its centre of mass can be found, are stored. From this information $\varepsilon(i, j)$ can be calculated based on the area occupied by the particles in cell $i, j$. Since the porosity is an important parameter which considerably influences the gas-phase motion, a detailed check for overlap was made in which multiple cell overlap was taken into account. However, the porosity calculated in this way is based on a twodimensional analysis which is inconsistent with the applied empiricism in the calculation of the drag force exerted on a particle (Section 2.5) and the interfacial friction (Section 3.2.2) since the relevant correlations are based on actual three-dimensional systems. To correct for this inconsistency the porosity calculated on area basis $\left(\varepsilon_{2 D}\right)$ was transformed to a three-dimensional porosity $\left(\varepsilon_{3 D}\right)$ using the following equation:

$$
\varepsilon_{3 D}=1-\frac{2}{\sqrt{\pi \sqrt{3}}}\left(1-\varepsilon_{2 D}\right)^{3 / 2} .
$$

This equation has been derived on the basis of a comparison between a hexagonal lattice (two-dimensional) and an FCC unit cube (three-dimensional) assuming equal interparticle distances. In order to account for the interfacial friction in the momentum conservation equation (43) for the gas phase, cellaveraged values of the solids $x$ - and $y$-velocities $\left(v_{x}\right.$ 
and $v_{y}$ ) are required. These values are obtained from the discrete particle model by taking a simple number averaged value of the velocities of the particles in the considered control element:

$$
\mathbf{v}_{\mathrm{av}}=\frac{\sum_{k}^{n_{\mathrm{tot}}} \mathbf{v}_{\boldsymbol{k}}}{n_{\mathrm{tot}}} .
$$

\subsection{Boundary conditions}

For the incorporation of the boundary conditions the code developed by Kuipers uses a flag matrix which allows boundary conditions to be specified for each single cell. A variety of boundary conditions can be applied by specification of the value of the cell flag $\mathrm{fl}(i, j)$ which is associated with the relevant boundary condition for cell $(i, j)$. The cell flags and the corresponding boundary conditions that are featured in the present code are listed in Table 1. The typical set of boundary conditions used in the simulations performed in this study is shown in Fig. 6. Boundary conditions had to be applied to the cell-averaged values, obtained from the discrete particle model, as well. In order to mimic a distributor plate the porosity in the influx cells $(\mathrm{fl}(i, j)=4)$ was set to a constant value of 0.4. For the porosity in an impermeable wall cell, the porosity of the adjacent cell was copied. As a boundary condition for the cell-averaged solids velocity, the normal components at impermeable wall cells were set equal to zero, which means that for the standard problem set-up (Fig. 6) $v_{x}$ at the side walls and $v_{y}$ at the upper and lower walls were set equal to zero.

\section{COMPUTATIONAL STRATEGY}

The model described in the previous sections has been implemented in the POGO-code which was

Table 1. Cell flags and corresponding cell types for the hydrodynamic equations

\begin{tabular}{ll}
\hline$f(i, j)$ & \multicolumn{1}{c}{ Cell type of cell $(i, j)$} \\
\hline 1 & Interior cell, no boundary conditions have to be specified \\
2 & Impermeable wall, free slip boundaries \\
3 & Impermeable wall, no slip boundaries \\
4 & Influx cell, velocities have to be specified \\
5 & Prescribed pressure cell, free slip boundaries \\
6 & Continuous outflow cell, free slip boundaries \\
7 & Corner cell, no boundary conditions have to be specified \\
\hline
\end{tabular}

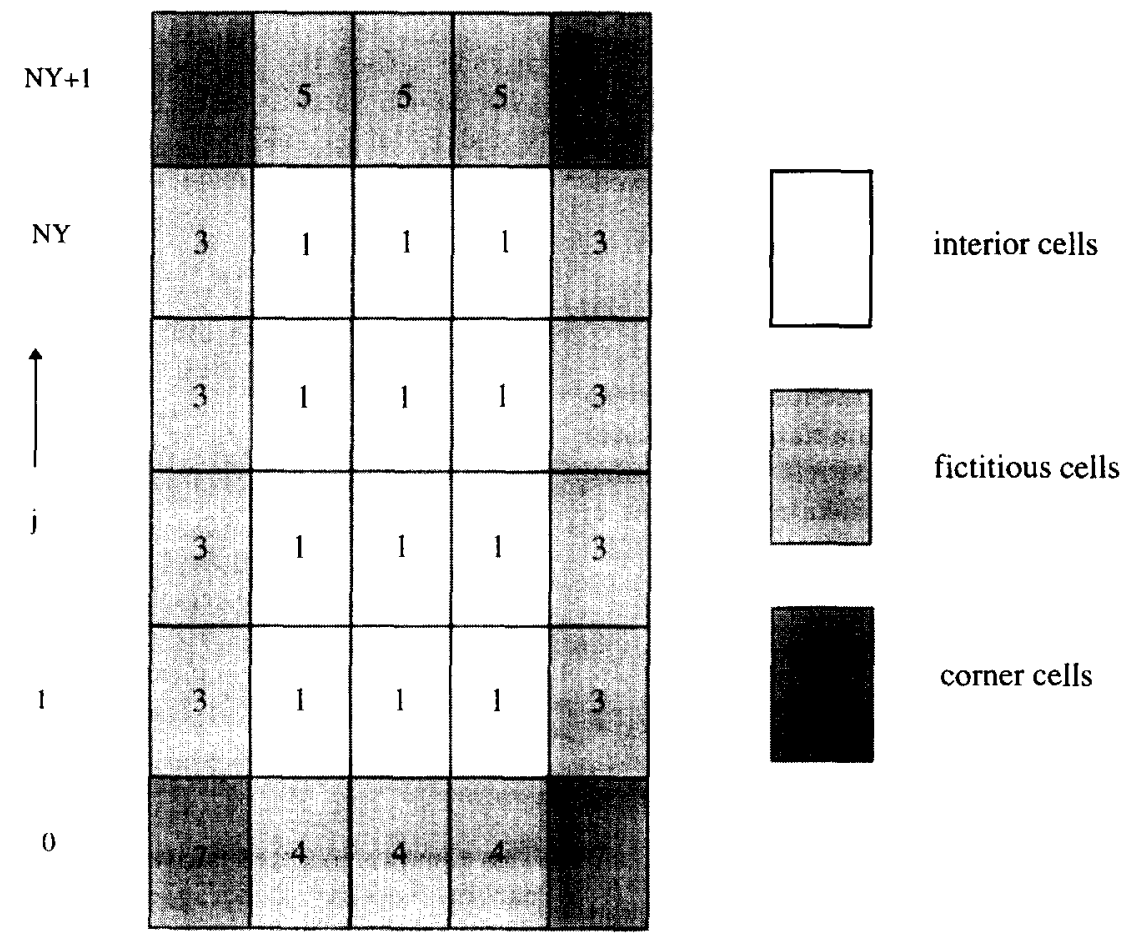

$0 \quad \mathrm{i} \quad \mathrm{i} \longrightarrow \mathrm{NX} \quad \mathrm{NX}+\mathrm{l}$

Fig 6. Schematic representation of the computational domain. 
written in ANSI-C. Its name is derived from four characteristic features of the code: Particles Obstacles Gas Orifice. The flowchart of the main routine in POGO is presented in Fig. 7. After initialisation the main loop is entered which is continued until the time $(t)$ exceeds the specified end-time (TSTOP). Within the loop, first the new particle velocities are calculated from the forces acting on a particle which is described in Section 2.5. With the new particle velocities a sequence of collisions is processed one collision at a time as explained in Section 2.4, where the dynamics of a single collision is calculated using the collision model presented in Section 2.3. At the end of this step the particle positions and velocities are known at the new time level. After calculation of the cell porosities and the cell-averaged solids velocities the gas flow field can be calculated by solving the Navier-Stokes equations which is described in Section 3. Finally the user-specified data is saved to file in the output manager and the main loop can be re-entered.

\subsection{Getting started}

Initial conditions were obtained by first placing the particles in a lattice configuration in the simulation box, causing a small disturbance and subsequently allowing them to fall under the influence of gravity in the absence of a gas flow field. As soon as a random particle configuration was established the particles were exposed to the influence of the gas motion. In the POGO-code the bed was set to minimum fluidisation conditions in order to obtain initial values for both the particle positions and velocities and the pressure and gas flow fields. This process could be monitored both visually, by plotting particle configurations, and analytically by plotting the potential energy of the system as a function of time. Once the potential energy started to fluctuate about an average value, the bed was assumed to be at the desired initial minimum fluidisation conditions.

\section{RESULTS AND DISCUSSION}

\subsection{Pouring into a container}

As a first test case for the discrete particle model motion in a vacuum was considered. The total amount of energy in such a system follows from the addition of the kinetic, potential and rotational energies of all particles and the energy dissipated in collisions (Section 2.3):

$$
E_{\mathrm{tot}}=E_{\mathrm{kin}}+E_{\mathrm{pot}}+E_{\mathrm{rot}}+E_{\mathrm{dsp}}
$$

and this amount should remain constant during a simulation. A series of simulations was performed in order to study the pouring into a container. Rosato et al. (1986) used a Monte Carlo technique in order to simulate the pouring into a container of 500 uniform disc-shaped particles. Hogue and Newland (1994) have repeated this simulation in order to validate their contact model. In these simulations 500 particles were randomly placed in a container of a 24 particles width and a 48 particles height. Our results of a simulation with the restitution coefficients $\left(e\right.$ and $e_{w}$ ) set

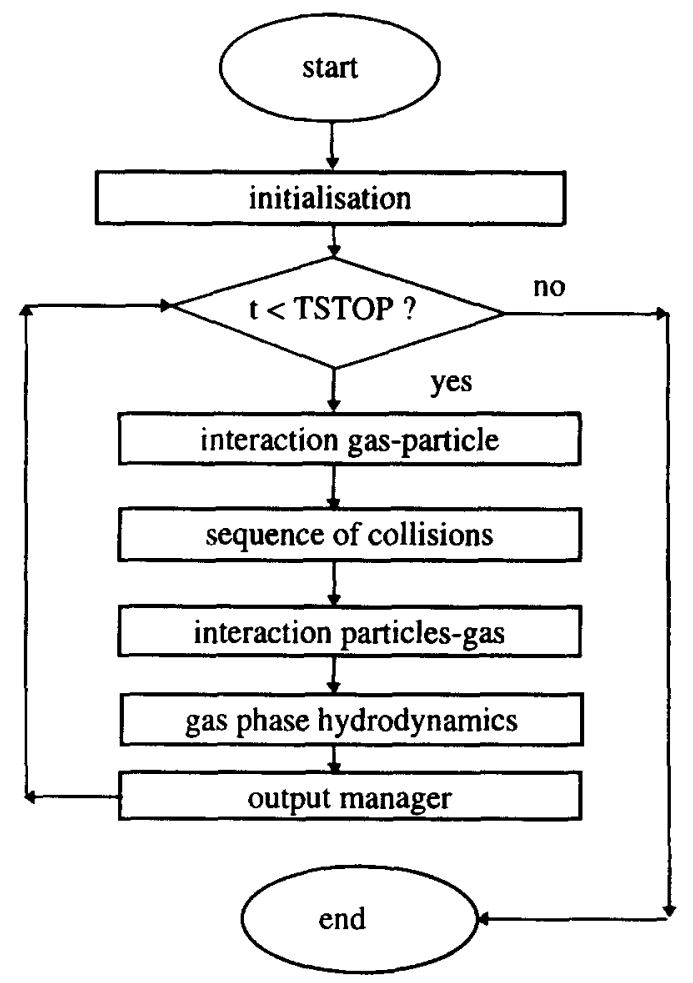

Fig 7. Computational strategy of the POGO-code.

equal to 0.9 and the friction coefficients $\left(\mu\right.$ and $\left.\mu_{w}\right)$ set equal to 0.3 are shown in Fig. 8. All the particle velocities were set equal to zero at the beginning of the simulation and the time step DT was chosen to be $10^{-4} \mathrm{~s}$. The energy housekeeping of the system is shown in Fig. 9 in which it can be seen that the contribution of the rotational energy to the total energy is negligibly small compared to the other contributions. Figure 9 shows clearly how potential energy is first converted into kinetic energy which in turn eventually is dissipated in collisions. The relative error in the energy conservation was about $0.005 \%$. This error was found to possess a first-order dependency with respect to the time step. The area fraction occupied by the particles at the end of the simulation was calculated by integrating the area under the upper layers of particles. A value of $0.832 \pm 0.010$ was found in this study, where Hogue and Newland (1994) found a value of 0.80 and Rosato et al. (1986) found a value of 0.805 . A comparison with the results presented by Berryman (1983) is not attempted here because those results are valid in the case of dense random packing. In the system simulated here, the packing cannot be classified as random because the system will tend to obtain the most favourable energetic situation due to gravity.

\subsection{The Tsuji simulations}

The only discrete particle model applied to fluidised beds so far was presented by Tsuji et al. (1993) who used the distinct element method (DEM) to represent the interparticle contact forces. The parameter settings they used in their simulations were chosen to 


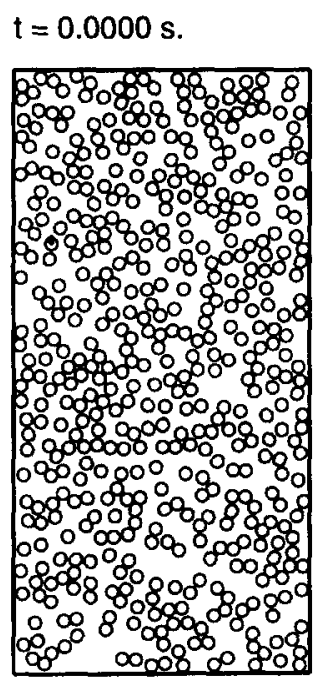

Fig 8. Snapshots of particle configurations for the pouring of 500 uniform particles into a container with $e=e_{w}=0.9$ and $\mu=\mu_{w}=0.3$.

energy $\mathrm{J} / \mathrm{kg}$

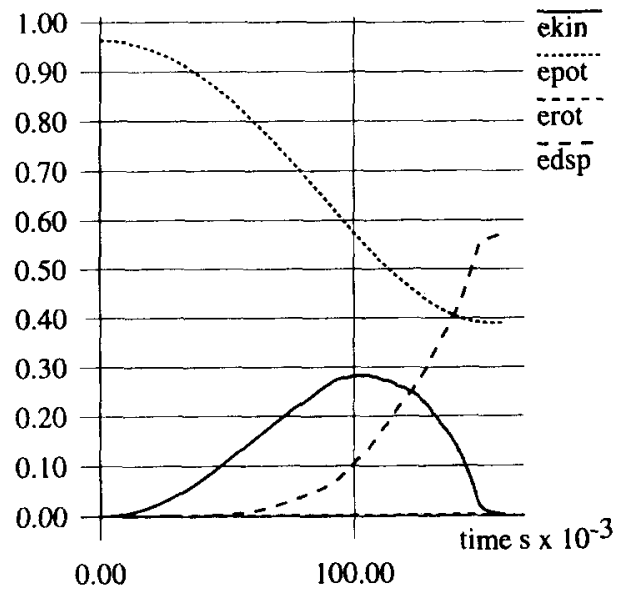

Fig 9. Total kinetic, potential, rotational and dissipated energy as a function of time for the pouring of 500 uniform particles into a container with $e=e_{w}=0.9$ and $\mu=\mu_{w}=0.3$. The error in the total conservation of energy was less than $0.005 \%$.

serve as a first test case for the POGO-code. The general parameter settings are listed in Table 2 . The minimum fluidisation velocity of the particles is 1.77 $\mathrm{m} \mathrm{s}^{-1}$ and the terminal velocity is $16.5 \mathrm{~m} \mathrm{~s}^{-1}$. First an attempt was made to reproduce the results of a simulation reported by Tsuji et al. (1993) where they found significant bubble formation when a superficial velocity of $2.6 \mathrm{~m} \mathrm{~s}^{-1}\left(<1.5 u_{m f}\right)$ in a central orifice was specified. In this work the superficial velocity in cell $(i, j)$ is defined to be the product of the porosity and the velocity in that cell. Since in this work the porosity in the bottom row of cells with $\mathrm{fl}(i, j)=4$ is set equal to 0.4 in order to mimic a distributor plate, this means that at the position of the orifice (bed centre) a velocity of $6.5 \mathrm{~m} \mathrm{~s}^{-1}$ was specified whereas in the other cells in that row a velocity of $4.5 \mathrm{~m} \mathrm{~s}^{-1}$ was specified
(1.8 $\mathrm{m} \mathrm{s}^{-1}$ superficial) as the background fluidisation velocity. It was not possible to reproduce the results of Tsuji et al. with the POGO-code. No bubble formation was observed in our simulation, only the porosity directly above the jet was slightly increased. This result was verified in an experiment using the experimental set-up described in Section 5.4 with $4 \mathrm{~mm}$ ballotini glass beads as the bed material taking into account the slightly higher minimum fluidisation velocity. In accordance with our simulation no bubble formation occurred at all. Since we do not know all the details of the Tsuji simulations (i.e. calculation of the drag force acting on the particle, calculation of the void fraction) we cannot say why our results differ from the results obtained by Tsuji et al (1993). However, we do not feel that the difference is caused by the DEM model employed in their model. It may be possible that the superficial velocity reported in their work refers to the bed width instead of the nozzle width.

\subsection{Sensitivity to model parameters}

The Tsuji set-up (Table 2) was subsequently used to perform a sensitivity analysis in order to study the influence of the two key parameters of the model: the restitution coefficient, $e$, and the friction coefficient, $\mu$ on bubble and slug formation. In all these simulations identical initial conditions were used. A time step of $10^{-4} \mathrm{~s}$ was used and the simulations were continued for one second real time.

5.3.1. Bubble formation at a single orifice. A series of simulations of bubble formation at a single, central orifice was performed. In the central cell of the bottom row a velocity of $45 \mathrm{~ms}^{-1}\left(10 u_{m f}\right)$ was specified which is equal to a superficial velocity of $18 \mathrm{~m} \mathrm{~s}^{-1}$ in that cell. In the other cells in the bottom row $4.5 \mathrm{~m} \mathrm{~s}^{-1}$ was specified as a background fluidisation velocity $\left(1.8 \mathrm{~m} \mathrm{~s}^{-1}\right.$ superficial). As can be seen from the snapshots shown in Fig. 10 the calculation is highly 
Table 2. Parameter settings for the Tsuji simulations

\begin{tabular}{llll}
\hline \multicolumn{1}{c}{ Particles } & \multicolumn{2}{c}{ Bed } \\
\hline Shape & spherical & Width & $150 \mathrm{~mm}$ \\
Diameter, $d_{p}$ & $4 \mathrm{~mm}$ & Height & $500 \mathrm{~mm}$ \\
Material & aluminium & Orifice diameter, $d_{o}$ & $10 \mathrm{~mm}$ \\
Density, $\rho$ & $2700 \mathrm{~kg} \mathrm{~m}^{-3}$ & Cell width, DX & $10 \mathrm{~mm}$ \\
$e, e_{w}$ & 0.9 & Cell height, DY & $20 \mathrm{~mm}$ \\
$\mu, \mu_{w}$ & 0.3 & Number $x$-cells, NX & 15 \\
Number & 2400 & Number $y$-cells, NY & 25 \\
\hline
\end{tabular}

(a)
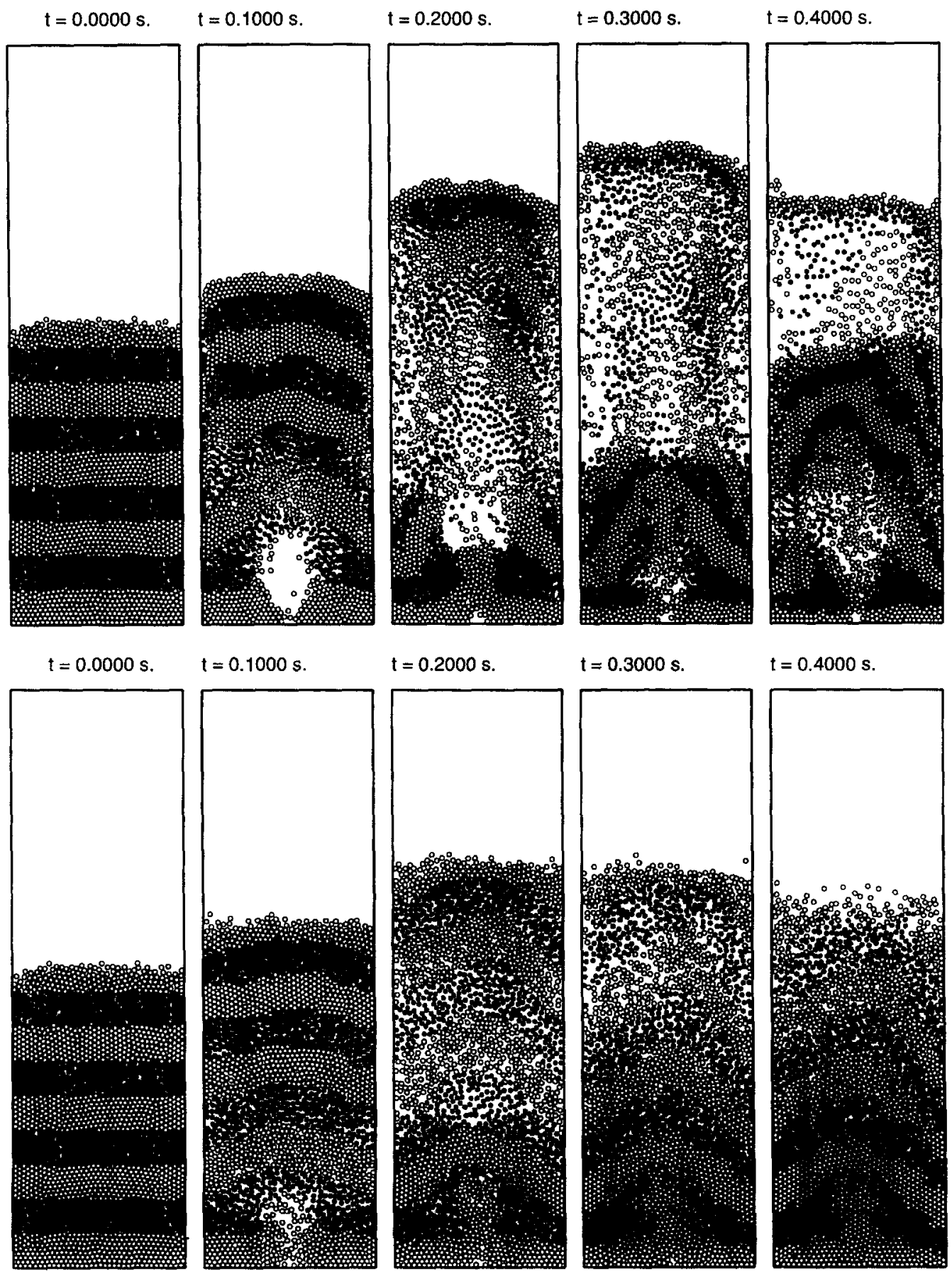

$t=0.3000$ s.

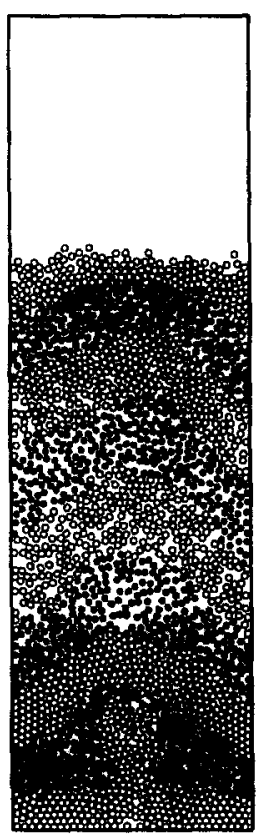

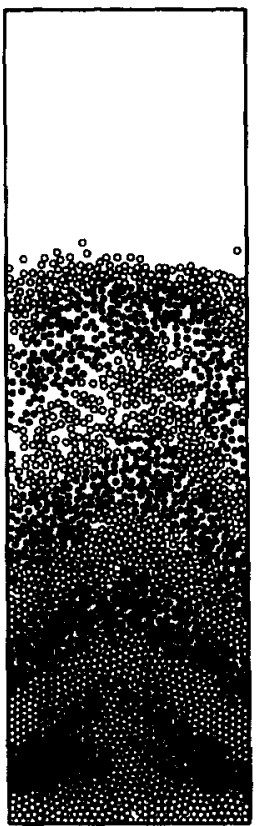

$t=0.4000 s$.

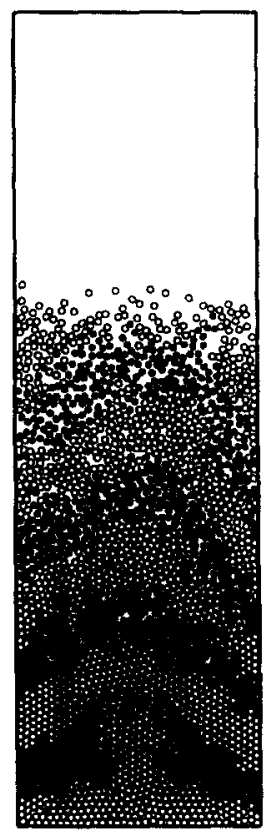

Fig 10. Snapshots of particle configurations for the simulation of bubble formation at a single orifice (jet velocity $10 u_{m f}$ ) in a two-dimensional bed (see Table 2.): (a), (c) $e=e_{w}=0.9, \mu=\mu_{w}=0.3$, (b), (d)

$$
e=e_{w}=1.0, \mu=\mu_{w}=0.0 \text {. }
$$


(c)
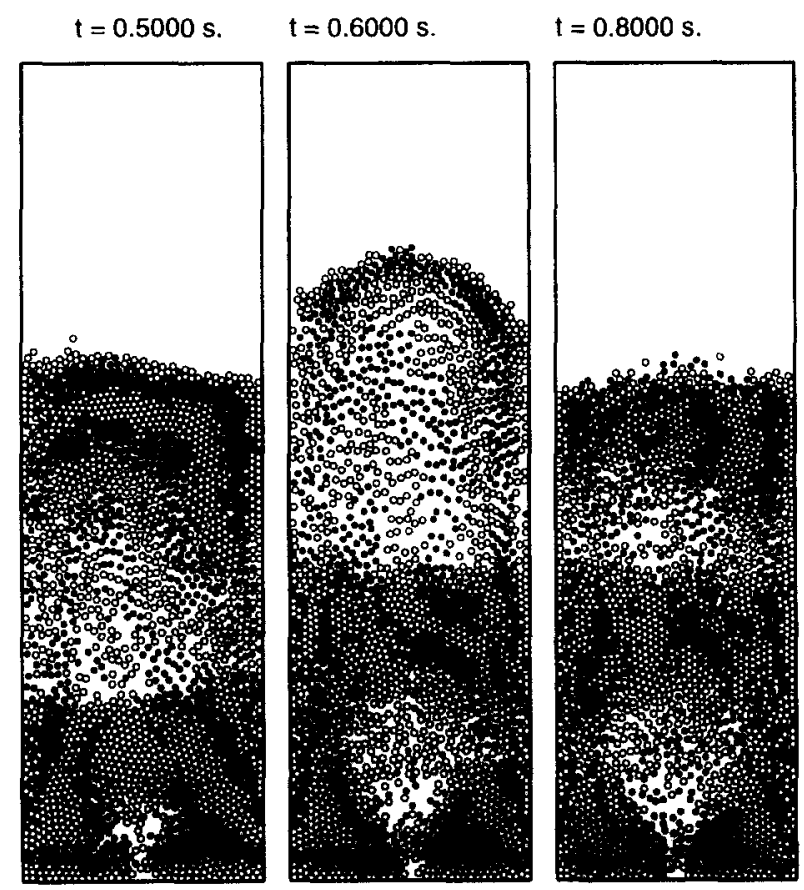

$t=1.0000$ s.
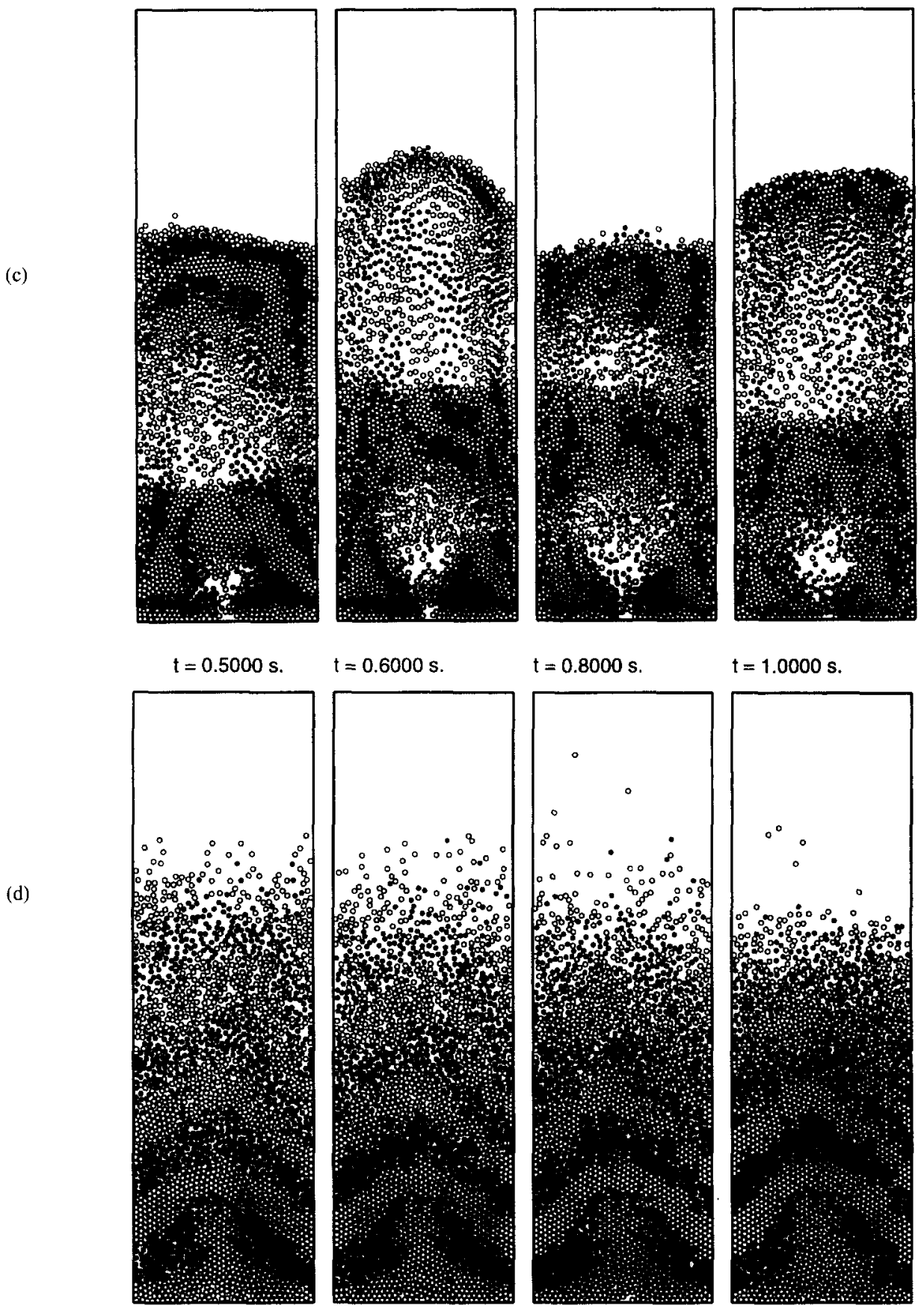

Fig. 10. (c) and (d).

sensitive to the restitution and friction coefficients. In the case of ideal particles $\left(e=e_{w}=1.0, \mu=\mu_{w}=0.0\right)$ no bubble formation can be observed and after $0.3 \mathrm{~s}$ the bed height remains relatively constant in time. In the simulation with more realistic particles ( $e=e_{w}=0.9, \mu=\mu_{w}=0.3$ ) explosive bubble growth can be observed which is expected behaviour for a $D$ powder. Initially a bubble is formed at the orifice but after a few centimetres of elevation the bubble transforms into a slug. All these characteristics are in accordance with experimental results. Note that the model predicts an asymmetrical bubble shape. In the same figure the effect on the mixing of the particles in the bed can be observed as well since certain layers of particles are marked. In animations of the simulations the circulatory motion of the particles (moving up at the centre and falling down at the sides) could clearly be observed. In Fig. 11, where the pressure $20 \mathrm{~cm}$ above the jet is plotted as a function of time, it can be seen that $e$ and $\mu$ have a strong influence on the gas-phase hydrodynamics as well. Simulations with intermediate values for $e$ and $\mu$ revealed that the effect 


$$
\text { pressure, } \mathrm{Pa} \times 10^{3}
$$

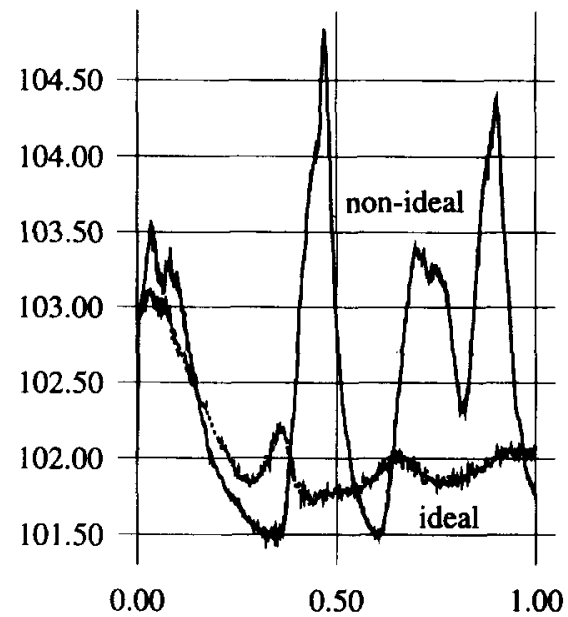

time, $s$.

Fig 11. Pressure in the bed, $20 \mathrm{~cm}$ above the jet, as a function of time for the simulation of bubble formation at a singie orifice (jet velocity $10 u_{m f}$ ) in a two-dimensional bed (see Table 2). The line with the strongest peaks, marked "nonideal", was obtained with $e=e_{w}=0.9, \mu=\mu_{w}=0.3$, the line marked "ideal" was obtained with $e=e_{w}=1.0, \mu=\mu_{w}=0.0$.

was a matter of degree whilst $e$ and $\mu$ have a larger influence than $e_{w}$ and $\mu_{w}$.

The effect of the restitution and friction coefficients can be explained as follows. Kinetic and rotational energy of the particles is dissipated in collisions if non-ideal particles are modelled. This means that the velocities after impact will be smaller than in the ideal case which leads to a particle configuration where the particles are more closely spaced which results in a lower value for the porosity. The drag force exerted on the particles depends strongly on the porosity: the lower the porosity the higher the drag force. Hence the drag force exerted on the particles in a closely spaced ensemble is high enough to overcome gravity and the particles will move upward. At the bottom region of a bubble or slug the porosity is higher which corresponds to a smaller drag force. This allows gravity to take over again and the particles start to rain off the bubble or slug roof.

5.3.2. Slug formation with homogeneous inflow conditions. Two simulations were performed with the POGO-code in order to simulate slug flow using the Tsuji set-up (Table 2) and the same initial conditions used in the bubble formation simulations. In the bottom row of cells with flag $\mathrm{f}(i, 0)=4$ a uniform velocity of $7.5 \mathrm{~m} \mathrm{~s}^{-1}$ was specified $\left(3.0 \mathrm{~m} \mathrm{~s}^{-1}\right.$ superficial, $1.75 u_{m}$ ). Snapshots of the particle configurations during both simulations are shown in Fig. 12. The influence of the two parameters can clearly be observed: in the case of ideal particles no slugging occurred at all and the bed expanded homogeneously which is not expected for this type of powder. The simulation with non-ideal particles clearly shows the model capability of predicting the formation slugs and bubbles starting from homogeneous inflow conditions. These predictions are rather unique in fluidised bed modelling. In Fig. 13 the pressure $20 \mathrm{~cm}$ above the jet is plotted as a function of time. Note that the simulations with the realistic values for the coefficients of restitution and friction show the characteristic pressure peaks due to the propagation of the slugs.

In the simulation with ideal particles slightly over one million collisions occurred during the transient whereas in the simulation with non-ideal particles over three million collisions occurred. Such a simulation takes less than $2 \mathrm{~h}$ of full priority computing on a Silicon Graphics Indigo 2 workstation.

\subsection{Experimental validation}

The POGO-code was validated experimentally using the experimental set-up which is shown schematically in Fig. 14 (Nieuwland, 1994). The primary fluidising gas was fed to the bed through two equally sized distribution chambers. From these distribution chambers the primary fluidisation gas was introduced into the fluidised bed section through a porous sintered stainless steel plate with a mean pore diameter of $10 \mu \mathrm{m}$. This porous plate, which served as the main distributor of the primary fluidising gas, was provided with a central rectangular pipe (internal dimensions: $15.0 \times 15.0 \mathrm{~mm}$ ) to inject secondary fluidising gas. This rectangular jet was covered with a piece of stainless steel gauze to prevent the particles from entering it. During the experiments three different gas flow rates were adjusted independently by means of calibrated thermal mass flow controllers: a gas flow through the distributor section to keep the bed at incipient fluidisation conditions, a gas flow through the rectangular orifice to fluidise the particles above the orifice and a gas flow for the bubble formation at the orifice. Prior to bubble injection, the secondary gas was purged, while the primary gas flowed through the gas distributor and the orifice to keep the bed at minimum fluidisation conditions. Rapidly responding magnetic valves, controlled by a microcomputer, were used to inject secondary gas through the orifice into the bed to form a bubble and to purge the primary gas during the period of injection. In the experiment the primary gas was introduced at a superficial velocity of $0.5 \mathrm{~m} \mathrm{~s}^{-1}\left(u_{m f}\right)$ and during a period of $200 \mathrm{~ms}$ secondary gas was injected through the central orifice at a superficial velocity of $2.5 \mathrm{~m} \mathrm{~s}^{-1}$. A S-VHS video camera was used to observe the bed during the processes of bubble formation and bubble growth. The bed material consisted of spherical ballotini glass particles with diameter $850 \pm 50 \mu \mathrm{m}$ and the density of the particle material was $2930 \mathrm{~kg} \mathrm{~m}^{-3}$. The minimum fluidisation velocity of $850 \mu \mathrm{m}$ ballotini is $0.49 \mathrm{~m} \mathrm{~s}^{-1}$. A simulation was performed with the POGO-code using the parameter settings as given in Table 3 . The values of the restitution and friction coefficients were obtained from simple bouncing experiments where single particle collisions were observed using a S-VHS video camera. For the determination of $e$ and $\mu$ ballotini particles were melted in order to produce a 
(a)

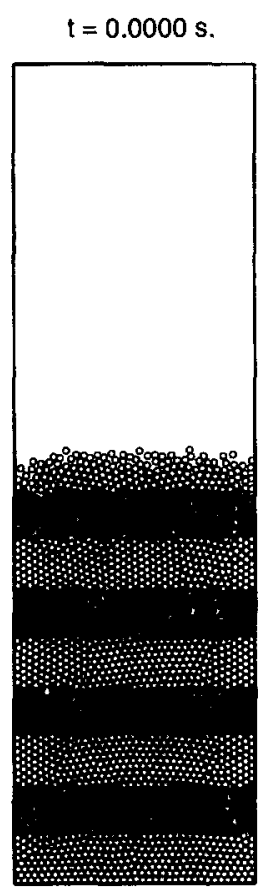

$t=0.1000 s$

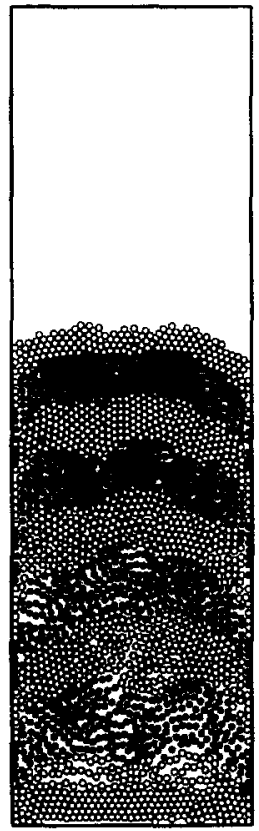

$t=0.0000 s$

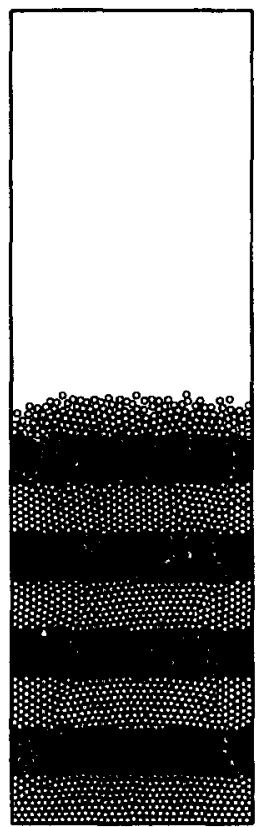

$t=0.2000 \mathrm{~s}$

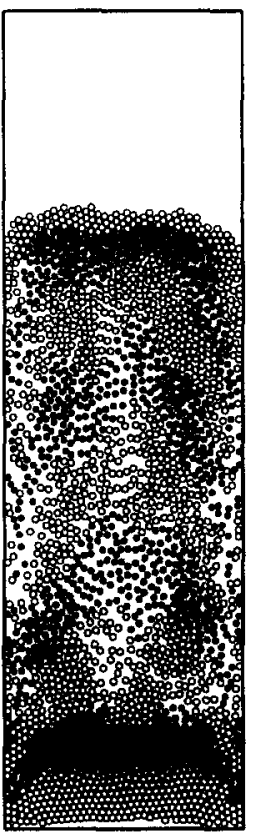

$t=0.2000 s$.

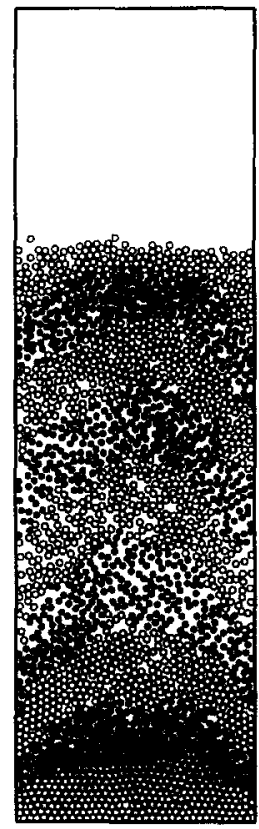

$t=0.3000 \mathrm{~s}$

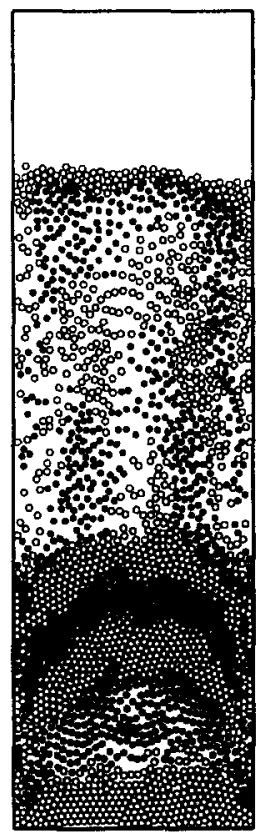

$t=0.3000 s$

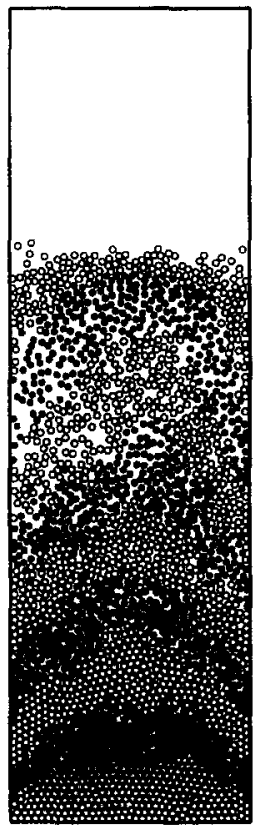

$t=0.4000 \mathrm{~s}$.

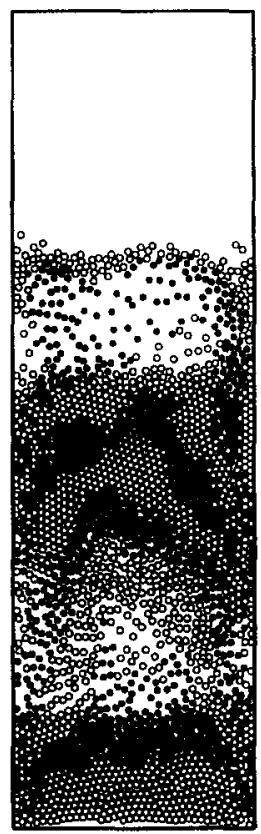

$t=0.4000 \mathrm{~s}$

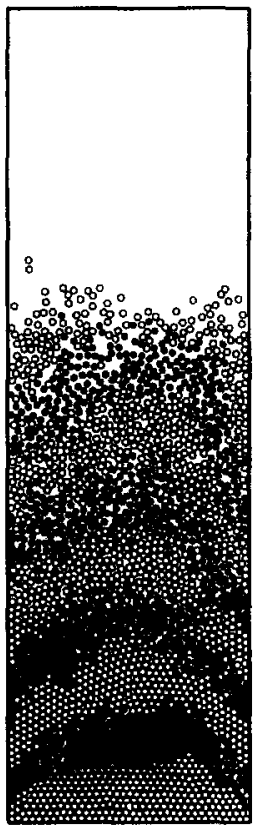

Fig 12. Snapshots of particle configurations for the simulation of slug formation with homogeneous inflow conditions $\left(1.75 u_{m f}\right)$ in a two-dimensional bed (see Table 2.): (a), (c) $e=e_{w}=0.9, \mu=\mu_{w}=0.3$, (b), (d)

$$
e=e_{w}=1.0, \mu=\mu_{w}=0.0 .
$$


(c)
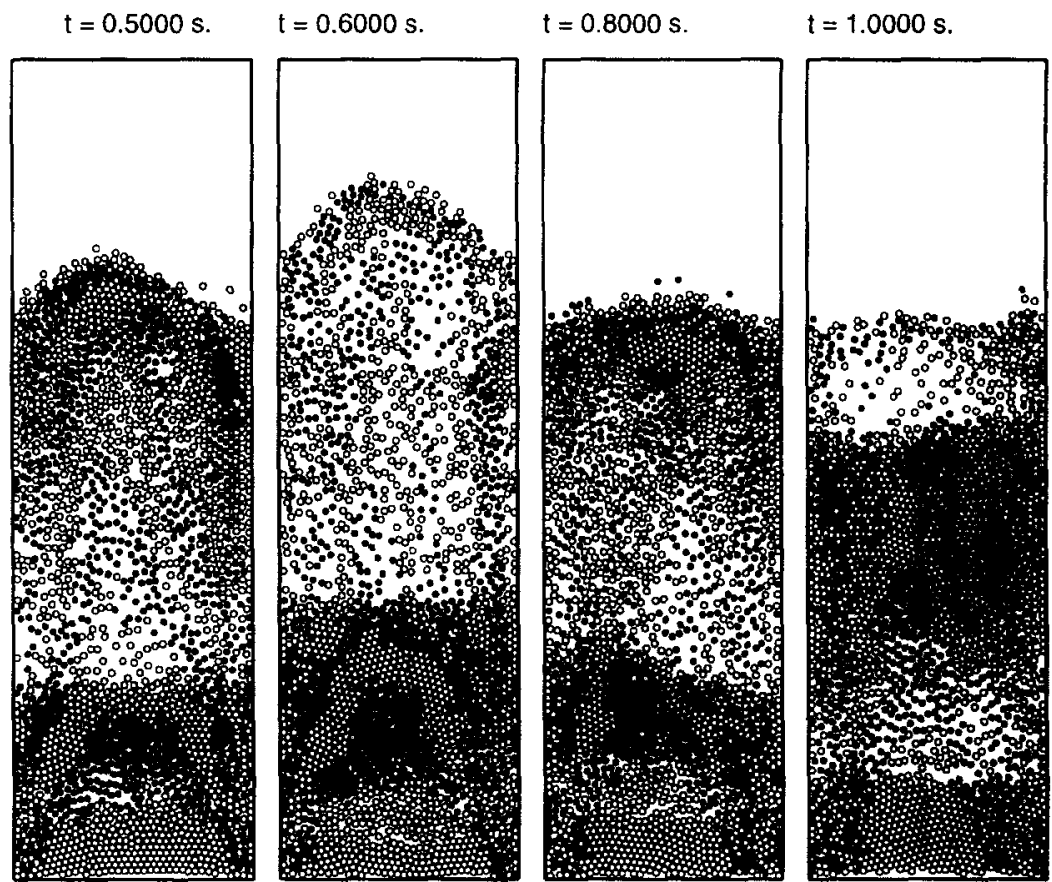

(d)

$t=0.5000 \mathrm{~s}$.

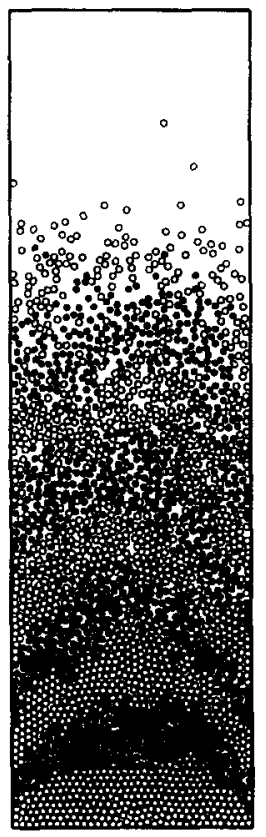

$t=0.6000 \mathrm{~s}$.

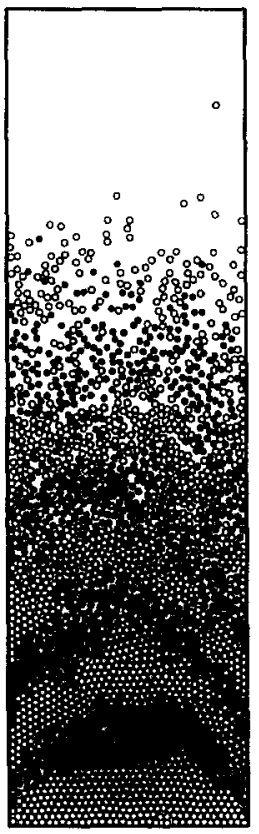

$t=0.8000 \mathrm{~s}$.

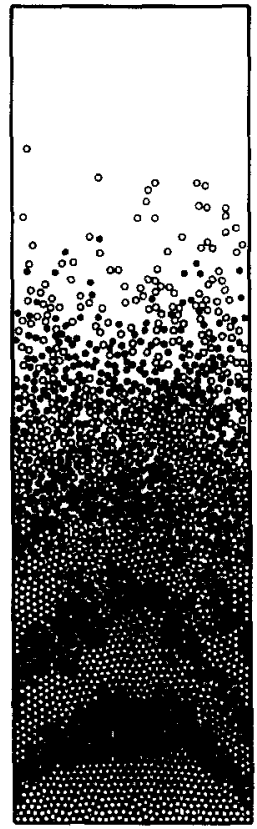

$t=1.0000 \mathrm{~s}$

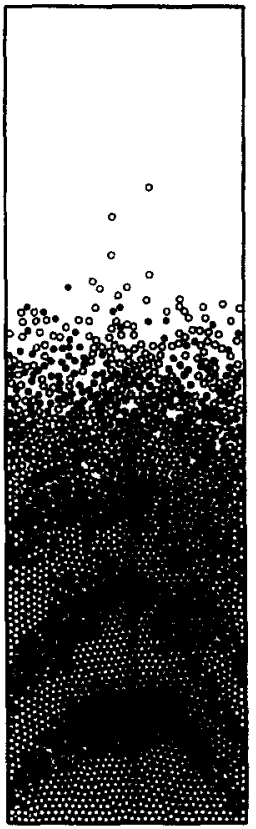

Fig. 12. (c) and (d). 
pressure, $\operatorname{Pa} \times 10^{3}$

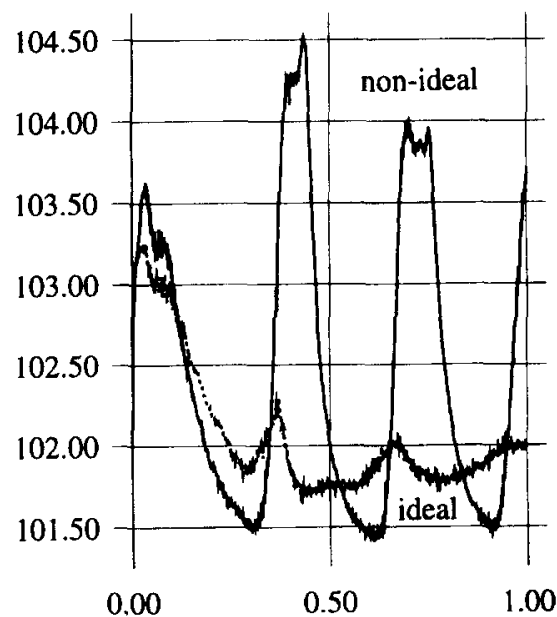

Fig 13. Pressure in the bed, $20 \mathrm{~cm}$ above the jet, as a function of time for the simulation of slug formation with homogeneous inflow conditions $\left(1.75 u_{m f}\right)$ in a two-dimensional bed (see Table 2). The line with the strongest peaks, marked "non-ideal", was obtained with $e=e_{w}=0.9, \mu=\mu_{w}=0.3$, the line marked "ideal" was obtained with $e=e_{w}=1.0$, $\mu=\mu_{w}=0.0$. ballotini glass plate. The wall material (Pyrex glass) was readily available in a plate form.

Results of the simulation are shown together with the experimentally obtained results in Fig. 15. The results show a reasonable agreement. The bubble that was observed experimentally tends to be smaller and it possesses sharper boundaries as well. The satellite bubbles that precede the main bubble in the simulation were not observed in the experiment. The difference is believed to be due to the two-dimensional nature of the simulation. The bed used in the experiment was still some 16 particle layers deep which means that our model is lacking an important degree of freedom for the motion of the particles. This lacking of the third dimension may have a significant effect when simulating the direct particle motion. Furthermore there was a particle size distribution $(850 \pm 50$ $\mu \mathrm{m})$ in the experiment which was not taken into account in the simulation. It is possible to improve agreement by artificially decreasing the coefficient of restitution, but since it is the objective of this work to predict the hydrodynamics of a fluidised bed from first principles, this tuning was not attempted. In the near future the present two-dimensional model will be extended to a full three-dimensional version which will

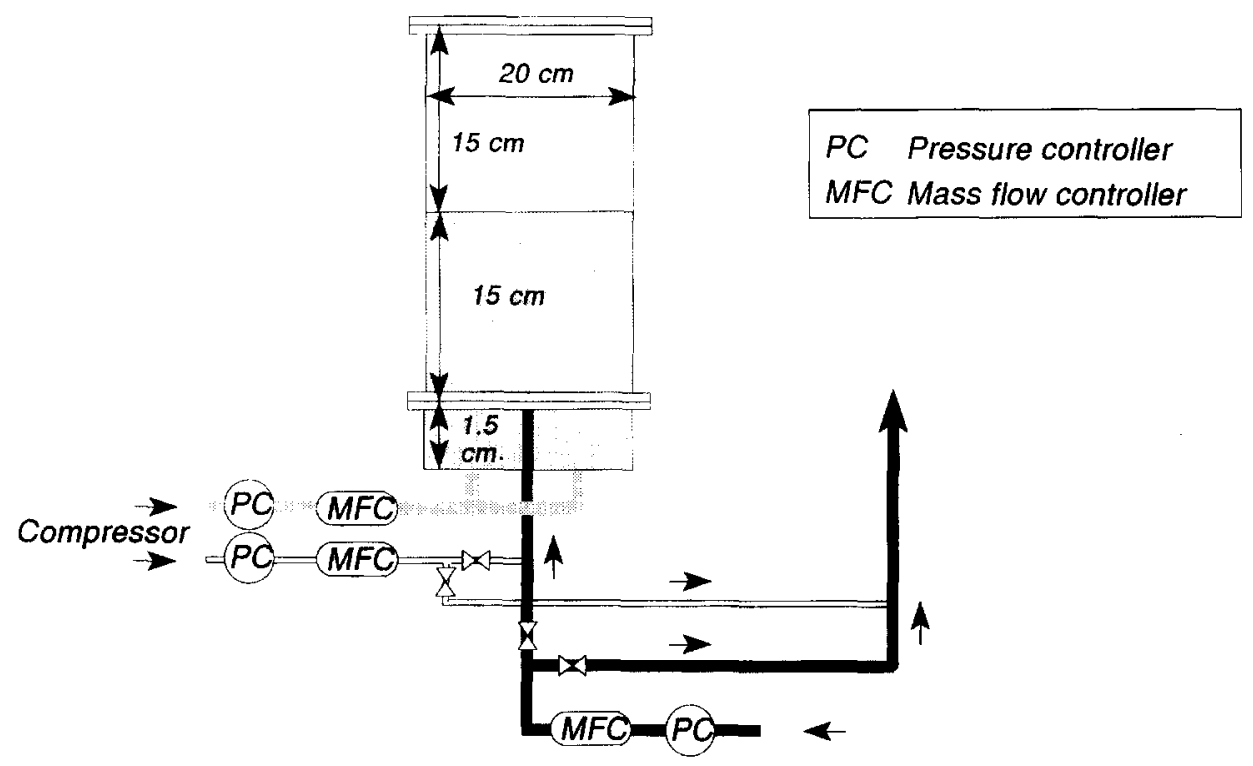

Fig. 14. Schematic representation of the experimental set-up.

Table 3. Parameter settings for the experimental validation

\begin{tabular}{llll}
\hline \multicolumn{1}{c}{ Particles } & \multicolumn{2}{c}{ Bed } \\
\hline Shape & spherical & Width & $195 \mathrm{~mm}$ \\
Diameter, $d_{p}$ & $850 \mu \mathrm{m}$ & Height & $300 \mathrm{~mm}$ \\
Material & ballotini glass & Orifice diameter, $d_{o}$ & $15 \mathrm{~mm}$ \\
Density, $\rho$ & $2930 \mathrm{~kg} \mathrm{~m}^{-3}$ & Cell width, DX & $5.0 \mathrm{~mm}$ \\
$e$ & 0.96 & Cell height, DY & $5.0 \mathrm{~mm}$ \\
$e^{\prime \prime}$ & 0.86 & Number $x$-cells, NX & 39 \\
$\mu$ & 0.15 & Number $y$-cells, NY & 60 \\
$\mu_{w}$ & 0.15 & & $2.5 \times 10^{-4} \mathrm{~s}$ \\
Number & 40,000 & Time step, DT & 2.0 \\
\hline
\end{tabular}


$t=0.0500 s$.
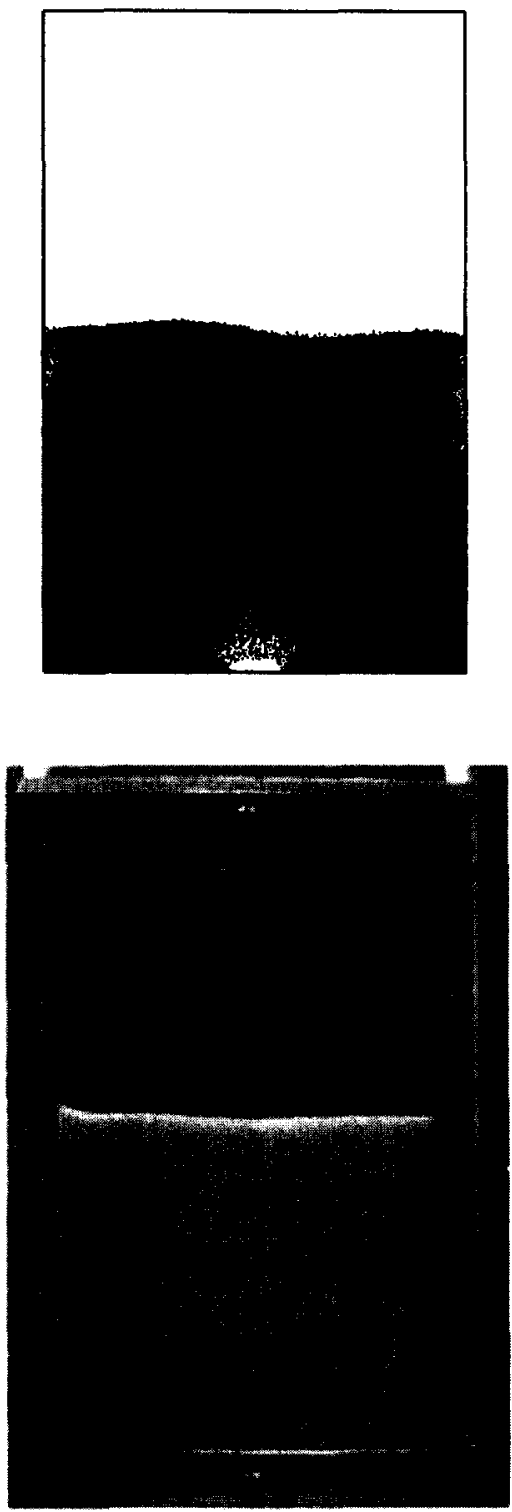

(a) $t=0.1000 \mathrm{~s}$.
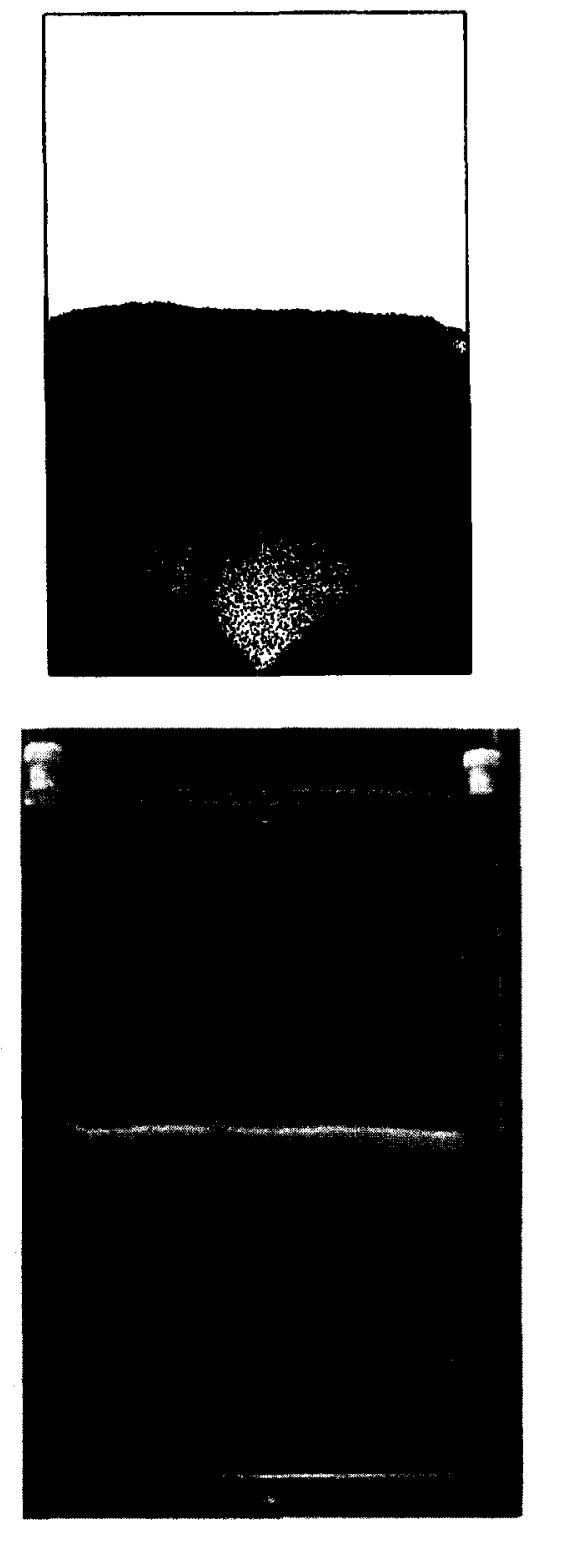

Fig. 15. (a) Comparison between experimental results and the results of a simulation (see Table 3) of bubble formation at a single orifice (jet velocity $5 u_{m f}$ ). 
$t=0.1500 \mathrm{~s}$.
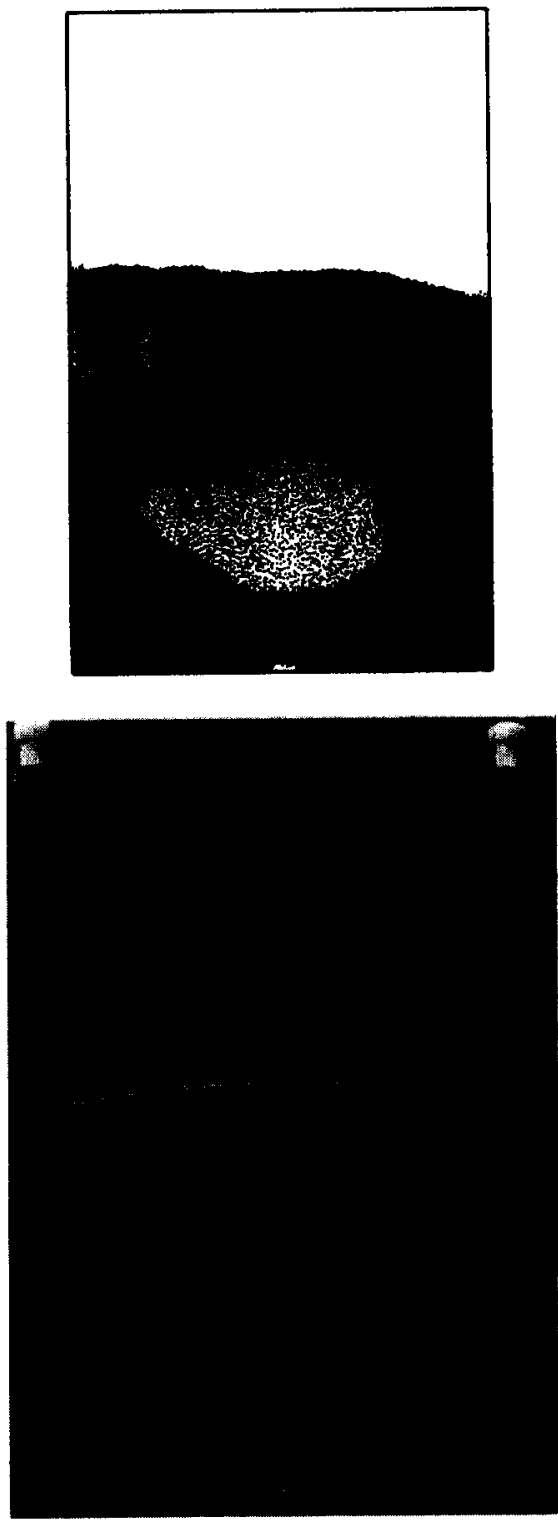

(b) $\mathrm{t}=0.2000 \mathrm{~s}$.
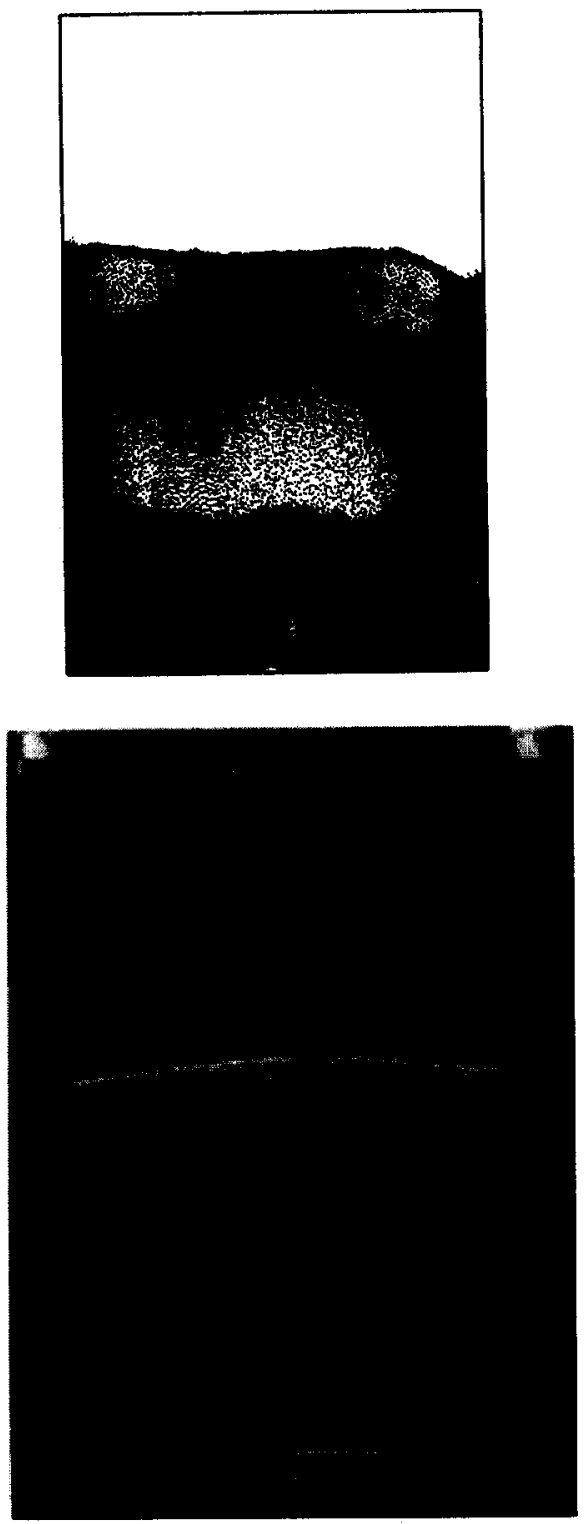

Fig. 15. (b) 
allow for a more detailed analysis of the aforementioned effects. A three-dimensional version of the hydrodynamic model of the gas phase has already been developed. The extension to three dimensions of the discrete particle model requires a more sophisticated collision model and the gridmapping techniques will be more complex as well. Implementation of a particle size distribution will be more straightforward since the model is in principle already capable of handling particles of different sizes.

\section{CONCLUSIONS}

A discrete particle model of a gas-fluidised bed has been developed in which the two-dimensional motion of the individual, spherical particles was directly calculated. A sequence of inelastic collisions with friction is processed one collision at a time. The hydrodynamic model of the gas phase is based on the Navier-Stokes equations for gas-solid two-phase flow. The collision model was shown to be energetically consistent. An attempt was made to repeat a simulation with 2400 particles reported by Tsuji et al. (1993) who found significant bubble formation when gas was injected through a central orifice at less than $1.5 u_{m f}$. It was not possible to reproduce the results presented by Tsuji et al. with our simulations. An independent experiment confirmed that no bubble formation occurred, contrary to the results reported by Tsuji et al.

A sensitivity analysis was performed in which the sensitivity of the results of the model to its key parameters (restitution and friction coefficients $e$ and $\mu$ ) was studied. The simulations were found to depend strongly on the values of $e$ and $\mu$. When ideal particles ( $e=1, \mu=0)$ were modelled the flow behaviour was found to be unrealistic which leads to the conclusion that it is of utmost importance that, in a model that requires a restitution and/or a friction coefficient, these parameters are given realistic values. Simulations with realistic values for $e$ and $\mu$ showed highly realistic flow behaviour for D-powder material. Explosive bubble growth followed by a transition to slug flow, which is rather typical for D-powder fluidisation, was predicted by the model when bubble formation at a single orifice was simulated. Our model was capable of predicting the formation of slugs when homogenous inflow conditions above minimum fluidisation conditions were specified.

Finally the model was validated experimentally in an experiment using $850 \mu \mathrm{m}$ ballotini glass beads in a small two-dimensional cold flow set-up described by Nieuwland (1994). The results of this experiment compared fairly well with the results of a simulation that was performed with 40,000 particles with realistic values for restitution and friction coefficients.

\begin{tabular}{ll} 
& \multicolumn{1}{c}{ NOTATION } \\
$A$ & area, $\mathrm{m}^{2}$ \\
$B_{1}, B_{2}$ & collision constants, $\mathrm{kg}^{-1}$ \\
$C_{d}$ & drag coefficient, dimensionless
\end{tabular}

\begin{tabular}{|c|c|}
\hline C & compression velocity, $\mathrm{m} \mathrm{s}^{-1}$ \\
\hline$D_{\text {nblist }}$ & diameter of neighbour square, $\mathrm{m}$ \\
\hline$d_{o}$ & orifice diameter, $\mathrm{m}$ \\
\hline$d_{p}$ & particle diameter, $\mathrm{m}$ \\
\hline $\begin{array}{l}d t_{n b l i s t} \\
\text { DT }\end{array}$ & $\begin{array}{l}\text { time step for neighbour list update, } s \\
\text { time step, } s\end{array}$ \\
\hline DX & horizontal computational cell dimension, $\mathrm{m}$ \\
\hline DY & vertical computational cell dimension, $\mathrm{m}$ \\
\hline$e$ & coefficient of restitution, dimensionless \\
\hline $\mathrm{E}$ & energy, $J$ \\
\hline $\mathrm{fl}(i, j)$ & cell flag, dimensionless, defined in Table 1 \\
\hline$F$ & force, $\mathrm{kg} \mathrm{m} \mathrm{s}^{-2}$ \\
\hline $\mathbf{g}$ & gravitational acceleration, $\mathrm{m} \mathrm{s}^{-2}$ \\
\hline$I$ & moment of inertia, $\mathrm{kg} \mathrm{m}^{2}$ \\
\hline 1 & unity tensor \\
\hline$i, j$ & cell indices, dimensionless \\
\hline$m$ & particle mass, $\mathrm{kg}$ \\
\hline$M$ & molecular weight, $\mathrm{kg} \mathrm{kmol}^{-1}$ \\
\hline NX & $\begin{array}{l}\text { number of computational cells in } x \text {-direc- } \\
\text { tion, dimensionless }\end{array}$ \\
\hline NYY & $\begin{array}{l}\text { number of computational cells in } y \text {-direc- } \\
\text { tion, dimensionless }\end{array}$ \\
\hline$n_{\text {tot }}$ & total number of particles, dimensionless \\
\hline$p$ & pressure, $\mathbf{P a}$ \\
\hline$P$ & accumulated impulse, $\mathrm{kg} \mathrm{m} \mathrm{s}^{-1}$ \\
\hline$Q$ & arbitrary quantity \\
\hline$R$ & gas constant, $\mathrm{J} \mathrm{mol}^{-1} \mathrm{~K}^{-1}$ \\
\hline$R_{p}$ & particle radius, $m$ \\
\hline $\mathbf{r}$ & position, $\mathrm{m}$ \\
\hline$s$ & sign [defined in eq. (17)], dimensionless \\
\hline$S$ & slip velocity, $\mathrm{m} \mathrm{s}^{-1}$ \\
\hline$t$ & time, $s$ \\
\hline$t_{a b}$ & collision time, $\mathrm{s}$ \\
\hline$T$ & temperature, $\mathbf{K}$ \\
\hline $\mathbf{u}$ & gas phase velocity, $\mathrm{m} \mathrm{s}^{-1}$ \\
\hline$u_{m f}$ & $\begin{array}{l}\text { superficial minimum fluidization velocity, } \\
\mathrm{m} \mathrm{s}^{-1}\end{array}$ \\
\hline 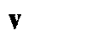 & velocity, $\mathrm{m} \mathrm{s}^{-1}$ \\
\hline$x$ & $x$-coordi \\
\hline
\end{tabular}

\section{Greek letters}

$\beta \quad$ volumetric interphase momentum transfer coefficient, $\mathrm{kg} \mathrm{m}^{-3} \mathrm{~s}^{-1}$

$\delta \quad$ distance, $m$

$\varepsilon \quad$ porosity, dimensionless

$\varepsilon_{m f} \quad$ porosity at minimum fluidisation conditions, dimensionless

$\mu_{q} \quad$ gas shear viscosity, $\mathrm{kg} \mathrm{m}^{-1} \mathrm{~s}^{-1}$

$\mu \quad$ friction coefficient, dimensionless

$\rho \quad$ density, $\mathrm{kg} \mathrm{m}^{-3}$

$\boldsymbol{T}_{g} \quad$ gas-phase stress tensor, $\mathrm{kg} \mathrm{m} \mathrm{s}^{-2}$

$\omega$ angular velocity, $\mathrm{s}^{-1}$

$\begin{array}{ll}\text { Subscripts } \\ 0 & \text { initial condition } \\ a, b & \text { particle indices } \\ \text { av } & \text { average } \\ c p & \text { contact point } \\ d & \text { drag } \\ d s p & \text { dissipated } \\ g & \text { gas phase }\end{array}$




$\begin{array}{ll}\text { gyr } & \text { gyration } \\ i, j & \text { cell indices } \\ \text { kin } & \text { kinetic } \\ m f & \text { minimum fluidisation conditions } \\ \text { nblist } & \text { neighbour list } \\ p & \text { particle } \\ \text { pot } & \text { potential } \\ \text { rot } & \text { rotational } \\ s & \text { solids phase } \\ X, x & \text { x-component } \\ Y, y & y \text {-component } \\ w & \text { wall } \\ & \\ \text { Superscripts } \\ \text { * } & \text { end of collision period } \\ \text { ' } & \text { most recent } \\ T & \text { effective } \\ & \text { transposed } \\ \text { Abbreviations } \\ \text { 2D } & \text { two dimensions } \\ \text { 3D } & \text { three dimensions } \\ \text { GD } & \text { granular dynamics } \\ \text { MD } & \text { molecular dynamics }\end{array}$

\section{REFERENCES}

Allen, M. P. and Tildesley, D. J., 1990, Computer Simulations of Liquids. Oxford Science Publications, Oxford.

Berryman, J. G., 1983, Definition of dense random packing. In Advances in the Mechanics and the Flow of Granular Materials (Edited by M. Shahinpoor), pp. 1-15. Trans. Tech. Aedermannsdorf, Switzerland.

Bird, R. B., Stewart, W. E. and Lightfood, E. N., 1960, Transport Phenomena. Wiley, New York.

Buyevich, YU. A., 1994, Fluid dynamics of coarse dispersions. Chem. Engng Sci. 49, 1217.

Campbell, C. and Potapov, A. V., 1993, Recent applications of computer simulation to granular systems. In The first Nisshin Engineering Particle Technology International Seminar (Edited by Y. Tsuji ), 18-20 January, p. 27. Nisshin Engineering Co. Ltd.
Cundall, P. D. and Strack, O. D. L., 1979, A discrete numerical model for granular assemblies. Geotechnique 29, 47.

Ding, J. and Gidaspow, D., 1990, A bubbling fluidization model using kinetic theory of granular flow. A.I.Ch.E.J. 36, 523.

Geldart, D., 1973, Types of gas fluidization. Powder Technol. $7,285$.

Hogue, C. and Newland, D., 1994, Efficient computer simulation of moving granular particles. Powder Technol. 78, 51.

Kuipers, J. A. M., van Duin, K. J., van Beckum, F. P. H and van Swaaij, W. P. M., 1992, A numerical model of gasfluidized beds. Chem. Engng Sci. 47, 1913.

Kuipers, J. A. M., van Duin, K. J., van Beckum, F. P. H. and van Swaaij, W. P. M., 1993, Computer simulation of the hydrodynamics of a two-dimensional gas-fluidized bed. Comput. Chem. Engng 17, 839.

Langston, P. A., Tüzün, U. and Heyes, D. M., 1994, Continuous potential discrete particle simulations of stress and velocity fields in hoppers transition from fluid to granular flow. Chem. Engng Sci. 49, 1259.

Langston, P. A., Tüzün, U. and Heyes, D. M., 1995, Discrete element simulation of granular flow in $2 \mathrm{D}$ and $3 \mathrm{D}$ hoppers: dependence of discharge rate and wall stress on particle interactions. Chem. Engng Sci. 50, 967.

Nieuwland, J. J., 1994, Hydrodynamic modelling of gassolid two-phase flows. Ph.D. thesis, Twente University, The Netherlands.

Richardson, J. F. and Zaki, W. N., 1954, Sedimentation and fluidisation: part I. Trans. Inst. Chem. Engng 32, 35.

Rosato, A., Prinz, F., Standburg, K. J. and Swendsen, R., 1986, Monte Carlo simulation of particulate matter segregation. Powder Technol. 49, 59.

Rowe, P. N. and Henwood, G. A., 1961, Drag forces in a hydraulic model of a fluidised bed-part I. Trans. Instn Chem. Engrs 39, 43.

Tsuji, Y., Kawaguchi, T. and Tanaka, T., 1993, Discrete particle simulation of two dimensional fluidized bed. Powder Technol. 77, 79

Tsuji, Y., Tanaka, T. and Ishida, T., 1992, Lagrangian numerical simulation of plug flow of cohesionless particles in a horizontal pipe. Powder Technol. 71, 239.

Walton, O. R., 1984, Application of molecular dynamics to macroscopic particles. Int. J. Engng Sci. 22, 1097.

Wang, Y. and Mason, M. T., 1992, Two-dimensional rigidbody collisions with friction. J. Appl. Mech, 59, 635.

Wen, C. Y. and Yu, Y. H., 1966, Mechanics of fluidization. Chem. Engng Prog. Symp. Ser. 62 (62), 100. 